\title{
Mitochondrial genome variation and prostate cancer: a review of the mutational landscape and application to clinical management
}

\author{
Anton M.F. Kalsbeek ${ }^{1,2}$, Eva K.F. Chan ${ }^{1,2}$, Niall M. Corcoran ${ }^{3,4}$, Christopher M. \\ Hovens $^{3,4}$ and Vanessa M. Hayes ${ }^{1,2,5}$ \\ 1'Laboratory for Human Comparative and Prostate Cancer Genomics, Genomics and Epigenetics Division, Garvan Institute of \\ Medical Research, Darlinghurst, New South Wales, Australia \\ ${ }^{2}$ Medical Faculty, University of New South Wales, Randwick, New South Wales, Australia \\ ${ }^{3}$ Australian Prostate Cancer Research Centre Epworth, Richmond, Victoria, Australia \\ ${ }^{4}$ Departments of Urology and Surgery, Royal Melbourne Hospital, University of Melbourne, Melbourne, Victoria, Australia \\ ${ }^{5}$ Central Clinical School, University of Sydney, Camperdown, New South Wales, Australia
}

Correspondence to: Vanessa M. Hayes, email: v.hayes@garvan.org.au

Keywords: prostate cancer, mitochondrial genome, mtDNA variation, biomarkers

Received: January 11, 2017

Accepted: July 26, 2017

Published: August 04, 2017

Copyright: Kalsbeek et al. This is an open-access article distributed under the terms of the Creative Commons Attribution License 3.0 (CC BY 3.0), which permits unrestricted use, distribution, and reproduction in any medium, provided the original author and source are credited.

\section{ABSTRACT}

Prostate cancer is a genetic disease. While next generation sequencing has allowed for the emergence of molecular taxonomy, classification is restricted to the nuclear genome. Mutations within the maternally inherited mitochondrial genome are known to impact cancer pathogenesis, as a result of disturbances in energy metabolism and apoptosis. With a higher mutation rate, limited repair and increased copy number compared to the nuclear genome, the clinical relevance of mitochondrial DNA (mtDNA) variation requires deeper exploration. Here we provide a systematic review of the landscape of prostate cancer associated mtDNA variation. While the jury is still out on the association between inherited mtDNA variation and prostate cancer risk, we collate a total of $\mathbf{7 4 9}$ uniquely reported prostate cancer associated somatic mutations. Support exists for number of somatic events, extent of heteroplasmy, and rate of recurrence of mtDNA mutations, increasing with disease aggression. While, the predicted pathogenic impact for recurrent prostate cancer associated mutations appears negligible, evidence exists for carcinogenic mutations impacting the cytochrome c oxidase complex and regulating metastasis through elevated reactive oxygen species production. Due to a lack of lethal cohort analyses, we provide additional unpublished data for metastatic disease. Discussing the advantages of mtDNA as a prostate cancer biomarker, we provide a review of current progress of including elevated mtDNA levels, of a large somatic deletion, acquired tRNAs mutations, heteroplasmy and total number of somatic events (mutational load). We confirm via meta-analysis a significant association between mtDNA mutational load and pathological staging at diagnosis or surgery $(p<0.0001)$.

\section{INTRODUCTION}

Mutations in the mitochondrial genome (mtDNA) have been implicated in both the initiation and progression of cancer, including prostate cancer (PCa). Oncogenic mtDNA mutations are hypothesized to play a role in the metabolic shift, from oxidative phosphorylation
(OXPHOS) to aerobic glycolysis, referred to as the Warburg effect [1,2]. A dysregulation of these processes could cause a general instability of mitochondrial homeostasis, thus impacting other mitochondrial functions such as apoptosis and calcium homeostasis [3]. Multiple reviews have addressed the importance of mitochondrial function in cancers [4-7]. As cancer cells 
require functional mitochondria, it is assumed that a fine balance exists in the type, frequency and number of mtDNA mutations a cancer cell can tolerate. Additionally, the oncogenic impact of acquired mtDNA mutations will depend on any inherited or ancestral mtDNA variation. Each of these distinct categories will be introduced and discussed in further detail in relation to $\mathrm{PCa}$.

$\mathrm{PCa}$ is a genetic disease, which has permitted the discovery of clinically relevant genetic-based biomarkers. Although several genetic tests for improving PCa diagnosis and possible prognosis are commercially available (reviewed in [8]), the clinical benefits of these tests are limited. Largely focused on the nuclear genome, these tests include assessments of gene fusion status, PTEN loss, gene expression signatures or methylation signatures. In contrast, the maternally inherited mtDNA has largely been overlooked in genome-based $\mathrm{PCa}$ biomarker discovery efforts. While there is clear evidence for mitochondrial genomic changes in prostate and other cancers (reviewed in $[5,9]$ ), the extent and impact of this variation to inform clinical management requires thorough investigation. Therefore, we conducted a systematic literature review and meta-analysis to investigate not only the mutational landscape of the mitochondrial genome in $\mathrm{PCa}$, but also the relationship between mtDNA variation and $\mathrm{PCa}$ risk, diagnosis and outcomes. We will also discuss potential therapeutic implications and future focus areas within this emerging field.

\section{PROSTATE CANCER IS A GENETIC DISEASE}

There are as yet no significantly verified modifiable risk factors for the prevention or development of $\mathrm{PCa}$ [10]. Genetics is the major contributor with a reported $57 \%(95 \%$ CI of $51 \%-63 \%)$ heritability [11]. Specifically, $\mathrm{PCa}$ risk is 2.35 times higher than average with an affected father, and even higher for affected brothers, with a diagnosis rate of 3.14 times the population average [12]. There is also significant risk associated with African-American ancestry, with 1.70 and 2.5-fold greater incidence and mortality rates than Americans of European ancestry, respectively [13]. Genome Wide Association Studies (GWAS) have identified a total of $100 \mathrm{PCa}$ susceptibility alleles, with modest effect sizes (odds ratio $<1.5$ ), estimated to explain roughly $33 \%$ of familial risk in men of European descent [14-16]. These common variants (minor allele frequency $\geq 5 \%$ ) are largely located within gene-free regions. Fine-mapping has led to the identification of novel independent signals within roughly a quarter of the PCa susceptibility loci [17]. Rare deleterious mutations in highly penetrant genes, such as BRCA2 (breast cancer associated), and HOXB13 (homeobox B13), explain only a small fraction (less than $6 \%$ ) of the missing heritability [18].
Built on the backbone of inherited variability, tumor progression is driven by acquired genomic variation. Next generation sequencing has greatly advanced the ability to sub-classify PCa based on acquired genomic signatures. The most recent analysis of 333 primary prostate tumor exomes by The Cancer Genome Atlas (TCGA) consortium identified seven molecular PCa subtypes based on recurrent oncogenic drivers. Over half involve an ETS (erythroblast transformation-specific transcription factor), specifically ERG, ETV1, ETV4 or FL11, gene fusion event, with TMPRSS2 (coding for a transmembrane serine protease) as the most common fusion partner. Roughly half of the ETS fusion-negative tumors present with mutations in $S P O P$ (coding for Speckled-type POZ protein), FOXA1 (coding for Forkhead box A1) or IDH1 (coding for isocitrate dehydrogenase-1), leaving 26\% PCa unclassified [19]. Additional known recurrent genomic alterations include; (i) mutations within genes coding for the tumor suppressor tumor protein P53 (TP53), the mediator complex subunit 12 (MED12) and a cell cycle inhibitor (CDKN1B), (ii) deletions and loss of function mutations within PTEN (phosphatase and tensin homolog), (iii) overexpression of serine peptidase inhibitor, Kazal type 1 (SPINK1), (iv) somatic copy number alterations (SCNAs) frequently involving gains of chromosome 7 and $8 \mathrm{q}$ (including the $M Y C$ oncogene locus) and losses of chromosome $8 \mathrm{p}$ (including oncogenes FGFR1 and WHSC1L1), 13q, 16q and 18 , and (v) epigenetic changes, such as silencing of genes encoding Src homology 2 domain containing $\mathrm{F}(S H F)$, fatty acid hydroxylase domain containing 2 (FAXDC2), glutathione S-transferase pi 1 (GSTP1), zinc finger protein 154 (ZNF154), and Kruppel like factor 8 (KLF8), the latter reviewed in detail elsewhere [20]. Additionally, unlike other epithelial cancers, PCa shows an abundance of large genomic rearrangements in relation to small somatic variants, suggesting a decreased impact of persistent mutagenic exposure [21, 22].

While both inherited and acquired genomic variation has been significantly linked to $\mathrm{PCa}$, previous studies have focused primarily on the nuclear genome, largely ignoring the maternally inherited mitochondrial genome.

\section{THE MITOCHONDRIAL GENOME}

Mitochondria are organelles responsible for major cellular processes. These include: ATP production through oxidative phosphorylation (OXPHOS), generating reactive oxygen species (ROS), maintaining calcium homeostasis and controlling apoptosis. Each cell contains hundreds to thousands of mitochondria, each housing tens to hundreds of copies of the circular genome. The 16,569 bases of mtDNA encode 37 genes, including 13 essential proteins that are components of four complexes (I, III, IV, V) of the electron transport chain for OXPHOS and the RNA machinery for assembly of these proteins, two ribosomal 
RNAs (12S and 16S rRNAs) and 22 tRNAs (Figure 1A). The remaining $7 \%$ (1,121 bases) of mtDNA makes up the non-coding control region, also known as the displacement (D)-Loop, where transcription and replication of the mtDNA is initiated. The functional relevance of mtDNA variation can therefore not be ignored.

With efficient DNA-repair mechanisms and lack of protective histones, the mtDNA is prone to mutations, with an approximately 10-fold higher mutation rate than the nuclear genome [23]. The high mutation rate and multiple copies of mtDNA within a cell can lead to a state of heteroplasmy, where not all mitochondrial genomes in a cell or mitochondrion are the same. In contrast to homoplasmy, where all mtDNA molecules are identical, the co-existence of multiple clonal populations of different mitochondrial genomes may contribute significantly to associated clinical heterogeneity. For inherited mitochondrial diseases, it is speculated that the ratio of mutated to wild-type mtDNA required for tissues to reach disease status is $7: 3$, which is further dependent on both the type of mutation and gene impacted [24-26]. We'll discuss known mitochondrial mutations and their association with PCa in the following sections.

\section{INHERITED MITOCHONDRIAL GENOME VARIATION AND PCA}

The Prostate Cancer Database Sweden (PCBaSe) has provided substantial evidence that the probability of being diagnosed with PCa is $14.9 \%$ in men with one affected brother compared with only $4.8 \%$ in the general population [27]. This risk in men with an affected brother over an affected father [12] may suggest a link to the maternally inherited mtDNA. Lack of recombination and strict maternal inheritance of mtDNA, has resulted in the accumulation of inherited mutations defining populationspecific mtDNA profiles, known as haplogroups. It has been shown that the variants found in different haplogroups confer different metabolic profiles to cells, suggesting potential haplogroup specific cancer predisposition [28]. Based on demonstrated correlation between population sub-group and $\mathrm{PCa}$ risk, with AfricanAmericans having the highest, Europeans intermediate and Asians the lowest risks [29, 30], it is therefore reasonable to postulate that mtDNA haplogroups may provide a susceptibility marker for PCa.

An initial 2006 North American study $(n=221$ cases, 246 controls), suggested an increased PCa risk with a 1.95 odds ratio for the European-derived haplogroup $U$, present in $23 \%$ of controls and $37 \%$ of the PCa patients [31]. This was followed shortly thereafter by a U-haplogroup targeted study ( $n=71$ cases, 128 controls) supporting risk association [32], although later studies were unable to confirm this association (Table 1). While many of these studies were underpowered, a lack of association was also reported from larger studies, including a focused study of 24 European haplogroup defining mtDNA markers in roughly 1000 cases and 500 controls [33] and a mtDNA-wide European study of 620 cases and 616 controls [34]. Similarly, a recent multiethnic American study ( $n=4,086$ cases 3,698 controls), including an almost even distribution between African-, Asian-, European- and Latino-Americans, reported no association with haplogroup $U$ in Americans of European ancestry. In the same study, although a nominally significant difference in PCa risk for the Eurasian-derived haplogroup $\mathrm{N}$ was found, the investigators noted the false positive rate was greater than $20 \%$ after multiple testing correction [35].

The question that remains is whether increased PCa risk observed in men with African heritage, may be associated with the presentation of earlier derived mitochondrial haplogroups common within Africa. The earliest diverged haplogroups within Africa display the greatest within population and between sub-population mitochondrial genome variation [36]. One study, although limited in numbers $(n=87$, including 26 noncancer prostate samples) found that the earliest diverged mitochondrial haplogroup, L0, specifically LOd from southern Africa, presented with higher pathology grade PCa than those with more recently diverging (non-L0) haplogroups (mean Gleason Score 6.3 versus mean Gleason Score 4.9, $p=0.049$ ) [37]. Given this tantalizing result and the known ethnic disparity in $\mathrm{PCa}$, more indepth investigation seems warranted.

In addition to haplogroup-defining mutations, other population-based inherited mutations have also been implicated in PCa risk. In particular, a 2005 study suggested that $C O 1$ missense mutations, common within the general European population, increased a man's risk for $\mathrm{PCa}$ [38]. The most common include non-synonymous polymorphisms, $6253 \mathrm{~T}>\mathrm{C}(\mathrm{M} 117 \mathrm{~T}), 6261 \mathrm{G}>\mathrm{A}$ (A120T), $6340 \mathrm{C}>\mathrm{T}$ (T146I) and $6663 \mathrm{~A}>\mathrm{G}$ (I254V). A second study led by the same team, found no evidence that CO1 mutations play a role in PCa risk in African American men [37], while the previously mentioned multi-ethnic study conversely found the $6253 \mathrm{~T}>\mathrm{C}$ polymorphism to be nominally protective in 1,661 European and 2,007 African Americans [35]. To truly evaluate an association between mtDNA haplogroups and/or $\mathrm{CO} 1$ polymorphic mtDNA variation and $\mathrm{PCa}$ risk and/or outcomes requires significantly larger studies.

\section{TOTAL SOMATIC MITOCHONDRIAL GENOME BURDEN IN PCA}

Compared with the nuclear genome, the mitochondrial genome shows a 55 times higher mutation rate in PCa [39]. Utilizing the TCGA data resource of 1,675 tumors from 31 tumor types, considerable difference in the number of acquired mtDNA mutations per patient (ranging from 0 to $7,31.1 \%>2$ variants) and position 
Table 1: Studies addressing mitochondrial haplogroup associated with prostate cancer risk

\begin{tabular}{|c|c|c|c|c|c|c|c|c|c|c|c|}
\hline \multirow[b]{2}{*}{ Reference } & \multirow[b]{2}{*}{$\begin{array}{l}\text { Ethnic group: } \\
\text { Population }\end{array}$} & \multirow{2}{*}{$\begin{array}{c}\text { Case } \\
\text { No. }\end{array}$} & \multicolumn{2}{|c|}{ Control } & \multicolumn{2}{|c|}{ U Haplogroup } & \multicolumn{2}{|c|}{ N Haplogroup } & \multicolumn{3}{|c|}{ Largest Representative Haplogroup } \\
\hline & & & No. & $\begin{array}{l}\text { Excl. } \\
\text { PCa }\end{array}$ & OR $(95 \% \mathrm{CI})$ & Power $^{1}$ & OR $(95 \% \mathrm{CI})$ & Power $^{1}$ & ID & OR $(95 \% \mathrm{CI})$ & Power $^{1}$ \\
\hline $\begin{array}{l}\text { Booker et al. } \\
2006[31]^{2}\end{array}$ & European: American & 221 & 246 & No & $1.95(1.13-3.38)$ & $83 \%$ & - & - & $\mathrm{H}$ & $0.88(0.61-1.28)$ & $8 \%$ \\
\hline $\begin{array}{l}\text { Kim et al. } \\
2008 \text { [99] }\end{array}$ & Asian: Korean & 139 & 122 & Yes & - & NA & $0.95(0.58-1.57)$ & $5 \%$ & $\mathrm{D}$ & $0.96(0.58-1.6)$ & $5 \%$ \\
\hline $\begin{array}{l}\text { Mueller et al. } \\
2009 \text { [100] }\end{array}$ & European: Austrian & 304 & 278 & Yes & $0.94(0.61-1.45)$ & $6 \%$ & - & - & $\mathrm{H}$ & $1.14(0.82-1.59)$ & $10 \%$ \\
\hline $\begin{array}{l}\text { Alvarez-Cubero et al. } \\
2012 \text { [95] }\end{array}$ & European: Spanish & 239 & 150 & No & $1.13(0.64-1.99)$ & $8 \%$ & - & - & $\mathrm{H}$ & $1.13(0.75-1.71)$ & $7 \%$ \\
\hline $\begin{array}{l}\text { Cano et al. } \\
2014 \text { [101] }\end{array}$ & Multi-ethnic: Colombian & 168 & 140 & No & $1.11(0.25-5.02)$ & $5 \%$ & 0 & - & $\mathrm{A}$ & $0.96(0.61-1.51)$ & $5 \%$ \\
\hline $\begin{array}{l}\text { Fachal et al. } \\
2014 \text { [34] }\end{array}$ & European: Spanish & 620 & 616 & no & $0.98(0.76-1.28)$ & $5 \%$ & - & - & $\mathrm{HV}$ & $1.07(0.85-1.34)$ & $7 \%$ \\
\hline \multirow{6}{*}{$\begin{array}{l}\text { Giorgi et al. } \\
2016[35]\end{array}$} & Multi-ethnic: American & 4086 & 3698 & Yes & $1.02(0.95-1.12)^{*}$ & $3 \%$ & $0.90(0.83-0.98)^{*}$ & $19 \%$ & $\mathrm{~L}$ & $1^{*}$ & - \\
\hline & European & 1004 & 1003 & - & $1.07(0.89-1.28)^{*}$ & $1 \%$ & & - & $\mathrm{L}$ & $1^{*}$ & - \\
\hline & Asian & 1009 & 999 & - & - & - & $0.91(0.85-0.99)^{*}$ & $7 \%$ & $\mathrm{D}$ & $1^{*}$ & - \\
\hline & African-American & 836 & 825 & - & $0.97(0.91-1.04)^{*}$ & $13 \%$ & $0.85(0.74-0.98)^{*}$ & $3 \%$ & $\mathrm{H}$ & $-*$ & - \\
\hline & Latino & 1099 & 739 & - & $1.09(0.95-1.25)^{*}$ & $2 \%$ & - & - & A & $1^{*}$ & - \\
\hline & Native Hawaiian & 138 & 132 & - & - & - & - & - & B & $1 *$ & - \\
\hline
\end{tabular}

Abbreviations: PCa, prostate cancer; OR, Odds Ratio; CI, confidence interval.

${ }^{1}$ Power calculations performed using a genetic power calculator [102] assuming a population PCa prevalence of 0.000548 [103], using the haplogroup frequencies as reported in the studies. ${ }^{2} \mathrm{U}$-haplogroup targeted analysis for 71 cases and 128 controls (excluded for PCa) reported an association with $\mathrm{PCa}(\mathrm{OR}=2.75,95 \% \mathrm{CI} 1.2-6.3)$ [32].

*Values as reported by study.

of mutations was reported [40]. PCa had the largest total somatic mtDNA mutational burden after gastric and hepatocellular carcinoma, with a predominance of single nucleotide variants (SNVs) $(n=154)$ over smaller insertion and deletions (INDELs) $(n=15)$ identified in 80 prostate tumors. Interestingly, this study showed that somatic mtDNA mutations are more likely a direct result of mtDNA replication error, contrary to the expectation that mtDNA mutations predominately result from external mutagens such as ROS. Additionally, missense mutations appeared to be largely heteroplasmic, suggesting negative selection of functionally deleterious mtDNA mutations.

A review of the literature as at 23 December 2016 using the search terms "mitochondrial genome" and "prostate cancer" led to the identification of eight published studies that met the inclusion criteria for whole genome or coding region mtDNA sequencing in $\mathrm{PCa}$, including data deposited into the International Cancer Genome Consortium (ICGC) database (Figure 1B). Five of the studies performed tissue-normal matched whole mtDNA sequencing (mWGS) via targeted next generation sequencing or multi-fragment capillary sequencing (cap) in men from USA $(n=10)$ [41], Canada $(n$ =24) [42], Austria $(n=30)$ [43], South Africa $(n=87)$ [37] and Australia $(n=115)$ [44]. Three analyzed the mtDNA generated by whole exome sequencing (WES) $(93 \%$ coding region) or whole genome sequencing (WGS) in PCa patients from Austria $(n=55)$ [39] or as part of the international TCGA $(n=80)$ [40] and ICGC $(n=21)$ [45] sequencing efforts, respectively. While most studies focused on primary $\mathrm{PCa}$ tissue, the South African study included prostate tissue from men with benign prostate hyperplasia (BPH) $(n=26)$ [37] and the USA study included matched primary $(n=9)$ and both soft $(n=10)$ and bone metastatic tissue $(n=10)$ from the same patients. The eight studies collectively report a total of 635 acquired SNVs and 25 INDELs in 284 of 396 men (71.7\%), impacting a total of 680 nucleotide positions ( $4.2 \%$ of the mitochondrial genome).

The total patient mutational burden ranged between 0 and 7 with an average of 1.60 variants per patient for localized disease, which is comparable with other tumor types [40]. The single study to include non-PCa (largely $\mathrm{BPH}$ ) patient prostate samples, reports significantly fewer somatic variants, specifically 17 in 20 non-PCa patients ( 0.85 per patient). As the only study from an African ancestral population, this study also reported a higher than average mutational burden for primary $\mathrm{PCa}$, averaging 1.85 somatic mutations per patient [37]. The increased mutational burden could also be as a direct result of more aggressive disease presentation, with almost $70 \%$ of the participants presenting with a Gleason score of 8 or greater. The Arnold et al., 2015 study, is the only published report to address somatic mtDNA mutational burden in metastatic $\mathrm{PCa}$ [41]. Sequencing the genomes of 10 matched bone and soft tissue metastases, further matched to nine primary prostate tissues, they reported an average of 4.0 and 5.2 somatic mutations, per patient, in soft tissue and bone metastases, respectively. This increase in overall somatic mtDNA mutations relative to those observed in primary $\mathrm{PCa}$, suggests an increase in frequency of somatic mtDNA mutations with tumor progression.

To further assess the total genomic landscape and persistence of mtDNA in PCa metastasis, we analyzed unpublished mitochondrial genome data generated from a published WGS data of seven metastatic PCa patients 
[46]. This study included nine bone metastases (two patients with multiple metastases) and one neuroendocrine PCa metastasis, as well as three matched primaries and a matched recurrence. The somatic mtDNA mutational burden, with associated frequencies, is summarized in Table 2. Although a low level of mutational heteroplasmy was observed in two of the three primary tumors (two somatic events per patient), none appear to persist within the matched bone metastasis. While overall the acquired mtDNA mutational burden increases with metastasis in these patients, only three mutations approach homoplasmic levels. Further studies are required to determine the overall impact of mtDNA mutations on metastatic PCa.

To understand the distribution and therefore the implication of somatic mtDNA mutations in PCa, we classified all reported mutations into mtDNA genic categories. As such we found the regions most impacted are the D-Loop $(n=74)$, the rRNA encoding $R N R 2$ $(n=62)$, and the CO1 gene $(n=59)$ (Figure 1B). When corrected for region and gene length, two tRNAs are highly impacted, tRNA-Pro and -Ala, with 103 and 87 mutations per $\mathrm{Kb}$, respectively. This might be exaggerated due to over-correction for the short length of tRNA genes.
When grouped together, tRNAs have 26 mutations per Kb. Within the larger regions, the D-Loop remains the most frequently mutated region, followed by $\mathrm{CO} 3$, RNR2, $\mathrm{CO} 2$ and $C O 1$ ( $n=66,43,40,38$ and 37, respectively). All three encoded proteins are located in the highly conserved OXPHOS complex IV region. The significance of variants in this complex driving early prostate tumor evolution requires further investigation. Least affected regions after correcting for gene length are ATP8, ND6, and ATP6 ( $n=5,23$ and 24, respectively) and most other tRNAs, suggesting a potential negative selection for mutations in those genes during tumorigenesis.

One should be mindful that reported differences in number and distribution of somatic variants detected may, at least in part, be a result of different variant calling methods, detection thresholds and/or populations sourced. The lack of rRNA and noncoding variants in the Parr et al. 2006 study, for example, can be attributed to the lower sequencing coverage $(78 \%)$ and the noted lack of the $12 \mathrm{~S}$ and $16 \mathrm{~S}$ rRNA regions due to nuclear mitochondrial DNA segment (numts) co-amplification [42]. For the Lindberg et al., 2013 [39] and ICGC studies [45], data was generated from WGE data and therefore excluded the noncoding region, while the targeted multi-fragment capillary
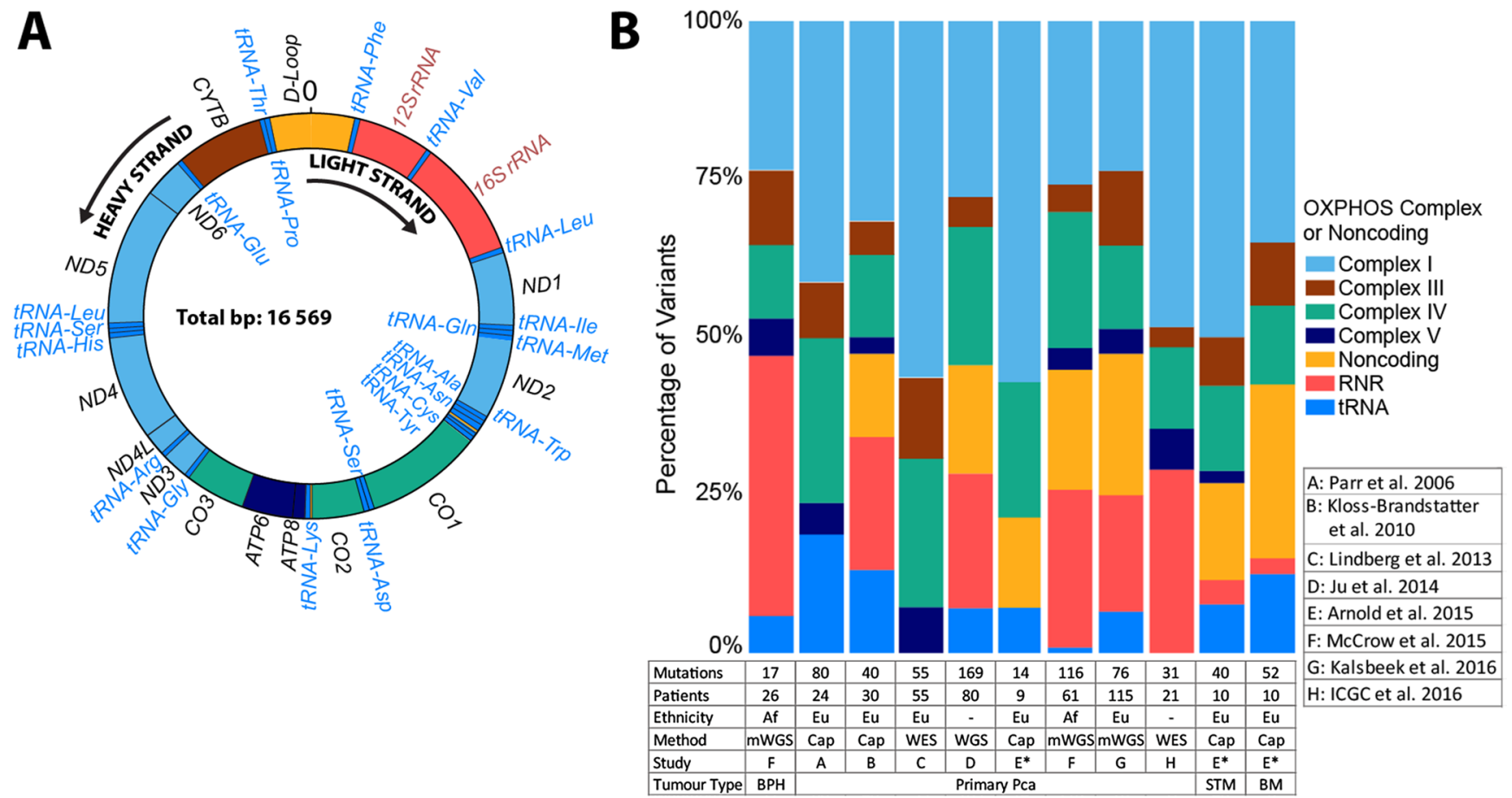

Figure 1: Mitochondrial genome schematic representation and distribution of prostate tissue somatic mutations colored by gene complexes. (A) Double stranded circular mitochondrial genome depicting the distribution and Heavy (H, outside) or Light (L, inside) strand direction of transcription for 37 genes, 22 tRNAs and 2 rRNAs. (B) Distribution of somatic mutations among the OXPHOS complexes and non-coding regions of the mitochondrial genome with numbers below each bar indicating the number of mutations and patients defined by ethnicity, specifically, European (Eu), or African (AF), where whole mitochondrial genome, either by whole genome sequencing (WGS), whole mitochondrial genome sequencing (mWGS) or via amplicon derived capillary sequencing (cap), or coding region analyses, include whole exome sequencing (WES) was performed, which includes a total of eight studies to date. Tissue types include predominantly primary $\mathrm{PCa}$, with smaller studies focused on benign prostate hyperplasia (BPH) or metastatic $\mathrm{PCa}$, specifically bone metastases (BM) or soft tissue metastasis (STM). A single study included patient matched primary and metastatic tissue $(*)$. 
Table 2: Somatic mtDNA mutations observed within metastatic tissue from seven men with prostate cancer derived from WGS data [46]

\begin{tabular}{|c|c|c|c|c|c|c|c|c|c|c|c|c|c|}
\hline \multirow[b]{2}{*}{ Patient } & \multirow[b]{2}{*}{ Metastasis } & \multirow[b]{2}{*}{ Position } & \multirow[b]{2}{*}{ Gene } & \multirow[b]{2}{*}{ Ref } & \multirow{2}{*}{\multicolumn{2}{|c|}{$\begin{array}{c}\text { Amino } \\
\text { Acid } \\
\text { change }\end{array}$}} & \multirow[b]{2}{*}{ Impact $^{1}$} & \multicolumn{6}{|c|}{ Alternate Allele Frequency $^{2}$} \\
\hline & & & & & & & & Primary & Recur & NEC & Bone1 & Bone2 & Bone3 \\
\hline \multirow{3}{*}{$\mathrm{SM}_{0} 02^{3}$} & \multirow{3}{*}{ Neuroendocrine } & 1301 & RNR1 & G & A & $\mathrm{NC}$ & $\mathrm{NC}$ & & & 0.32 & & & \\
\hline & & 6736 & COX1 & $\mathrm{T}$ & $\mathrm{C}$ & M278T & NS-Benign & & & 0.49 & & & \\
\hline & & 13019 & ND5 & G & A & G228D & NS-PD & & & 0.97 & & & \\
\hline \multirow{5}{*}{ SM001 } & \multirow{5}{*}{$\begin{array}{l}\text { Bone1: pubic } \\
\text { Bone2: pelvis }\end{array}$} & 10170 & ND3 & G & $\mathrm{C}$ & E38Q & NS-PD & & & & 0.86 & 0.80 & \\
\hline & & 16148 & D-Loop & $\mathrm{C}$ & $\mathrm{T}$ & $\mathrm{NC}$ & $\mathrm{NC}$ & & & & 0.82 & 0.90 & \\
\hline & & 2440 & RNR2 & G & A & $\mathrm{NC}$ & $\mathrm{NC}$ & & & & 0.19 & absent & \\
\hline & & 3492 & ND1 & A & $\mathrm{C}$ & $\mathrm{K} 62 \mathrm{~N}$ & NS-Benign & & & & 0.41 & absent & \\
\hline & & 8270 & Noncoding & $\mathrm{C}$ & $\begin{array}{c}\text { ins } \\
\text { CACCCCСТCT }\end{array}$ & $\mathrm{NC}$ & $\mathrm{NC}$ & & & & absent & 0.15 & \\
\hline SM067 & Bone1: spinal & 13513 & ND5 & G & $\mathrm{A}$ & D393N & NS-PD & & & & 0.40 & & \\
\hline SM068 & $\begin{array}{l}\text { Bone1: } \\
\text { vertebral }\end{array}$ & 316 & D-Loop & G & $\mathrm{C}$ & $\mathrm{NC}$ & $\mathrm{NC}$ & & & & 0.13 & & \\
\hline \multirow{3}{*}{ SM177 } & \multirow{3}{*}{ Bone1: ilium } & 567 & D-loop & A & $\mathrm{AC}$ & $\mathrm{NC}$ & $\mathrm{NC}$ & 0.28 & & & absent & & \\
\hline & & 6620 & COX1 & $\mathrm{T}$ & $\mathrm{C}$ & G239G & Syn & 0.26 & & & absent & & \\
\hline & & 8656 & ATP6 & A & $\mathrm{T}$ & $\mathrm{T} 44 \mathrm{~S}$ & Benign & absent & & & $0.61^{4}$ & & \\
\hline SM2995 & $\begin{array}{l}\text { Bone1: } \\
\text { shoulder }\end{array}$ & none & - & - & - & - & - & none & & & none & & \\
\hline \multirow{3}{*}{ SM498 } & Bone1: sacral & 3098 & RNR2 & $\mathrm{T}$ & $\mathrm{C}$ & $\mathrm{NC}$ & $\mathrm{NC}$ & 0.17 & absent & & - & absent & - \\
\hline & Bone2: ilium ${ }^{6}$ & 14849 & CYTB & $\mathrm{T}$ & $\mathrm{C}$ & S35P & NS-PD & 0.10 & absent & & absent & absent & absent \\
\hline & Bone3: ilium ${ }^{6}$ & 13198 & ND5 & G & A & $\mathrm{A} 288 \mathrm{~T}$ & Benign & absent & 0.38 & & 0.3 & 0.22 & 0.31 \\
\hline
\end{tabular}

Abbreviations: Ref, the revised Cambridge Reference Sequence (rCRS) allele; Alt, alternative (mutated) allele; Recur, local recurrence; NEC, neuroendocrine; NC, noncoding; NS, non-synonymous SNV; PD, probably damaging; Syn, Synonymous SNV.

Note: mtDNA sequence data was isolated from published WGS data from seven patients with 21 samples, including neuroendocrine $(n=1)$ or bone metastatic PCa $(n=9)$, with matched whole blood $(n=7)$ and primary PCa tissue $(n=3)$, with a single recurrence $(n=1)$ [46]. Average tumor purity was $61.6 \%$ (range $23 \%$ to $86 \%)$. Total mitochondrial genome coverage ranged from $885 \mathrm{X}$ to $11,403 \mathrm{X}$.

${ }^{1}$ Impact predicted using PolyPhen-2 [47]. ${ }^{2}$ Frequency of somatic mutations. ${ }^{3} \mathrm{~A}$ low frequency 0.18 heteroplasmic variant $6787 \mathrm{~T}>\mathrm{C}$ in $\mathrm{COI}$ gene was observed in the blood, however, this variant was absent in the matched neuroendocrine tissue from patient SM002. ${ }^{4}$ Mutation also observed in blood from patient SM177 but at lower frequency of $0.31 .{ }^{5}$ No somatic mutations were found in the mtDNA for patient SM299. ${ }^{6}$ Iliac bone metastasis sampled two months apart.

sequencing methods would each present with some level of amplification biases. Overall the WGS and mWGS studies report similar mutational distributions, with the single African study reporting an overall decrease in somatic mutations in the tRNAs compared to European studies.

\section{PCA ASSOCIATED MITOCHONDRIAL SOMATIC VARIANTS}

In addition to the eight whole genome and whole coding region mitochondrial studies for PCa mentioned above, additional studies include partial mtDNA analyses. Taken together, a total of 766 unique somatic mtDNA prostate tissue associated mutations have been identified to date (Supplementary Table 1). Excluding mutations in non-cancerous $\mathrm{BPH}$, the vast majority of the $749 \mathrm{PCa}-$ associated somatic mutations reported are defined as heteroplasmic, with only $14.8 \%$ (92/621 with reported heteroplasmy) described as homoplasmic. We note an increased frequency of metastatic PCa derived somatic mtDNA variations described as homoplasmic (46.2\%, 24/52) compared with primary PCa $(11.2 \%, 64 / 569)$. Although none of the non-synonymous homoplasmic mtDNA variants had a predicted damaging impact, using PolyPhen2 [47], their affect on ROS-production requires further investigation. It should be cautioned that all pathogenicity prediction tools have limitations, for example PolyPhen 2 is significantly better at detecting loss-of-function over gain-of-function mutations [48]. In our additional analyses, we identify three somatic mutations, two with predicted oncogenic potential, approaching homoplasmic levels in bone metastatic and brain metastatic neuroendocrine PCa (Table 2). Thus, the original observation that the level and degree of heteroplasmy increases with tumor activity, as based on semi-quantitative capillary sequencing data [43], may warrant further investigation.

Of all the somatic mtDNA mutations reported in $\mathrm{PCa}$, 80 sites $(9 \%)$ were observed in two or more patients (Table $3)$. Four of these, specifically 303, 16182, 16183 and 16519 , are known polymorphic sites and are therefore unlikely to have significant impact on $\mathrm{PCa}$ [49]. Of the remaining 76 sites, the most common include: $10398 \mathrm{~A}>\mathrm{G}, 72 \mathrm{~T}>\mathrm{C}$ and $11719 \mathrm{G}>\mathrm{A}$, presenting in seven, six and five patients, respectively. The next fourteen most common mutations were reported in three to four patients. Ten occur within the non-coding region, four are synonymous and the three non-synonymous mutations are predicted by PolyPhen 2 to be benign. Further evidence would be required, however, to support lack of pathogenicity. Factoring in the cautionary 
Table 3: Recurrent somatic mtDNA mutations reported for prostate cancer $(n=80$ nucleotide positions)

\begin{tabular}{|c|c|c|c|c|c|c|c|}
\hline 氮: & बृ & $\frac{i}{\frac{0}{0}}$ & 空. 童. & 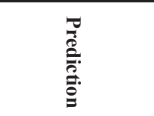 & 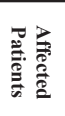 & $\begin{array}{l}\bar{\square} \\
\vdots \\
\overline{0}\end{array}$ & 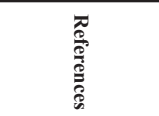 \\
\hline 10398 & ND3 & $\mathrm{A}>\mathrm{G}$ & T114A & NS-Benign & 7 & $\mathrm{BM}$ & [41] \\
\hline 72 & D-Loop & $\mathrm{T}>\mathrm{C}$ & $\mathrm{NC}$ & $\mathrm{NC}$ & 6 & $\mathrm{PPCa}$ & {$[104],[40],[37]$} \\
\hline 11719 & ND4 & $\mathrm{G}>\mathrm{A}$ & G320G & Syn & 5 & PPCa, BM, STM & [39], [41] \\
\hline $16519^{*}$ & D-Loop & $\mathrm{C}>\mathrm{C}, \mathrm{T}>\mathrm{C}$ & $\mathrm{NC}$ & $\mathrm{NC}$ & 5 & $\mathrm{PPCa}, \mathrm{BM}, \mathrm{STM}$ & $\begin{array}{c}{[105],[40],[41],} \\
{[44]}\end{array}$ \\
\hline 73 & D-Loop & $\mathrm{A}>\mathrm{G}$ & NC & NC & 4 & PPCa, BM & [105], [41] \\
\hline 195 & D-Loop & $\mathrm{C}>\mathrm{T}, \mathrm{T}>\mathrm{C}$ & NC & NC & 4 & PPCa, STM & [105], [44], [41] \\
\hline 9899 & $\mathrm{COX} 3$ & $\mathrm{C}>\mathrm{T}, \mathrm{T}>\mathrm{C}$ & $\mathrm{H} 231 \mathrm{H}$ & Syn, Syn & 4 & BM, STM & {$[41]$} \\
\hline 10463 & tRNA-Arg & $\mathrm{C}>\mathrm{T}, \mathrm{T}>\mathrm{C}$ & $\mathrm{NC}$ & $\mathrm{NC}$ & 4 & BM, STM & {$[41]$} \\
\hline 16093 & D-Loop & $\mathrm{C}>\mathrm{C}, \mathrm{C}>\mathrm{T}$ & $\mathrm{NC}$ & $\mathrm{NC}$ & 4 & $\mathrm{PPCa}$ & [105], [44] \\
\hline $16183^{*}$ & D-Loop & $\mathrm{A}>\mathrm{AC}, \mathrm{A}>\mathrm{C}, \mathrm{A}>\mathrm{G}$ & $\mathrm{NC}$ & $\mathrm{NC}$ & 4 & $\mathrm{PPCa}, \mathrm{BM}$ & $\begin{array}{c}{[90],[105],[40],} \\
{[46]}\end{array}$ \\
\hline 16189 & D-Loop & $\mathrm{T}>\mathrm{C}$ & $\mathrm{NC}$ & $\mathrm{NC}$ & 4 & $\mathrm{PPCa}$ & [90], [105], [40] \\
\hline $309 *$ & D-Loop & $8 \mathrm{C}>9 \mathrm{C}, 8 \mathrm{C}>7 \mathrm{C}, 7 \mathrm{C}>8 \mathrm{C}$ & $\mathrm{NC}$ & $\mathrm{NC}$ & 4 & $\mathrm{PPCa}$ & {$[43]$} \\
\hline 4216 & ND1 & $\mathrm{C}>\mathrm{T}, \mathrm{T}>\mathrm{C}, \mathrm{UN}$ & Y304H, H304Y, UN & NS-Benign, UN & 3 & PPCa, BM, STM & {$[42],[41]$} \\
\hline 11251 & ND4 & $\mathrm{A}>\mathrm{G}$ & L164L & $\mathrm{NC}$ & 3 & BM, STM & {$[41]$} \\
\hline 12372 & ND5 & $\mathrm{G}>\mathrm{A}$ & $\mathrm{L} 12 \mathrm{~L}$ & Syn & 3 & PPCa, STM & [90], [39], [41] \\
\hline 14766 & СYТВ & $\mathrm{C}>\mathrm{T}$ & T7I & NS-Benign & 3 & PPCa, BM, STM & [39], [41] \\
\hline 15928 & tRNA-Thr & $\mathrm{G}>\mathrm{A}$ & $\mathrm{NC}$ & $\mathrm{NC}$ & 3 & BM, STM & {$[41]$} \\
\hline 16294 & D-Loop & $\mathrm{C}>\mathrm{T}$ & $\mathrm{NC}$ & $\mathrm{NC}$ & 3 & PPCa, BM, STM & {$[41],[37]$} \\
\hline 16380 & D-Loop & $\mathrm{C}>\mathrm{T}$ & $\mathrm{NC}$ & $\mathrm{NC}$ & 3 & $\mathrm{PPCa}$ & {$[37]$} \\
\hline $303 *$ & D-Loop & $2 \mathrm{C}>\mathrm{C}, 8 \mathrm{C}>7 \mathrm{C}, 8 \mathrm{C}>9 \mathrm{C}, 2 \mathrm{C}>\mathrm{C}$ & $\mathrm{NC}$ & $\mathrm{NC}$ & 3 & $\mathrm{PPCa}, \mathrm{BM}$ & [105], [104], [46] \\
\hline 64 & D-Loop & $\mathrm{C}>\mathrm{A}$ & $\mathrm{NC}$ & $\mathrm{NC}$ & 2 & $\mathrm{PPCa}$ & {$[40],[37]$} \\
\hline 146 & D-Loop & $\mathrm{T}>\mathrm{C}$ & $\mathrm{NC}$ & $\mathrm{NC}$ & 2 & $\mathrm{PPCa}$ & {$[90],[40]$} \\
\hline 150 & D-Loop & $\mathrm{C}>\mathrm{T}$ & $\mathrm{NC}$ & $\mathrm{NC}$ & 2 & PPCa, BM & {$[105],[41]$} \\
\hline 152 & D-Loop & $\mathrm{C}>\mathrm{T}, \mathrm{T}>\mathrm{C}$ & $\mathrm{NC}$ & $\mathrm{NC}$ & 2 & $\mathrm{PPCa}, \mathrm{BM}$ & {$[41]$} \\
\hline 185 & D-Loop & $\mathrm{G}>\mathrm{A}, \mathrm{A}>\mathrm{A}$ & $\mathrm{NC}$ & $\mathrm{NC}$ & 2 & $\mathrm{PPCa}$ & [37], [44] \\
\hline 189 & D-Loop & $\mathrm{A}>\mathrm{G}$ & $\mathrm{NC}$ & $\mathrm{NC}$ & 2 & $\mathrm{PPCa}$ & [90], [37] \\
\hline 204 & D-Loop & $\mathrm{T}>\mathrm{C}$ & $\mathrm{NC}$ & $\mathrm{NC}$ & 2 & $\mathrm{PPCa}$ & {$[90],[40]$} \\
\hline 207 & D-Loop & $\mathrm{G}>\mathrm{A}$ & $\mathrm{NC}$ & $\mathrm{NC}$ & 2 & $\mathrm{PPCa}$ & [90], [105] \\
\hline 214 & D-Loop & $\mathrm{A}>\mathrm{G}$ & $\mathrm{NC}$ & $\mathrm{NC}$ & 2 & $\mathrm{PPCa}$ & [40], [37] \\
\hline 235 & D-Loop & $\mathrm{G}>\mathrm{C}, \mathrm{A}>\mathrm{G}$ & $\mathrm{NC}$ & NC & 2 & $\mathrm{PPCa}$ & {$[90],[105]$} \\
\hline 244 & D-Loop & $\mathrm{AA}>\mathrm{A}$ & $\mathrm{NC}$ & $\mathrm{NC}$ & 2 & $\mathrm{PPCa}$ & {$[105]$} \\
\hline 709 & RNR1 & $\mathrm{G}>\mathrm{A}$ & $\mathrm{NC}$ & rRNA & 2 & PPCa & [43], [37] \\
\hline 902 & RNR1 & $\mathrm{G}>\mathrm{A}$ & $\mathrm{NC}$ & rRNA & 2 & $\mathrm{PPCa}$ & [40], [37] \\
\hline 1339 & RNR1 & $\mathrm{G}>\mathrm{A}$ & $\mathrm{NC}$ & rRNA & 2 & PPCa & [40], [44] \\
\hline 1464 & RNR1 & $\mathrm{G}>\mathrm{A}$ & $\mathrm{NC}$ & rRNA & 2 & PPCa, STM & [41], [44] \\
\hline 1806 & RNR2 & $\mathrm{T}>\mathrm{C}$ & $\mathrm{NC}$ & rRNA & 2 & $\mathrm{PPCa}$ & {$[40]$} \\
\hline 2819 & RNR2 & $\mathrm{G}>\mathrm{A}$ & $\mathrm{NC}$ & rRNA & 2 & $\mathrm{PPCa}$ & {$[40]$} \\
\hline 3357 & ND1 & $\mathrm{G}>\mathrm{A}$ & M17M & Syn & 2 & $\mathrm{PPCa}$ & [90], [37] \\
\hline 3394 & ND1 & $\mathrm{T}>\mathrm{C}, \mathrm{UN}$ & Y30H, UN & NS-Benign, UN & 2 & $\mathrm{PPCa}$ & {$[42],[43]$} \\
\hline 3454 & ND1 & $\mathrm{G}>\mathrm{A}$ & A50T & NS-PD & 2 & $\mathrm{PPCa}$ & [40], [37] \\
\hline 3664 & ND1 & $\mathrm{G}>\mathrm{A}$ & G120X & Stopgain & 2 & PPCa & {$[40]$} \\
\hline 3915 & ND1 & $\mathrm{G}>\mathrm{A}$ & G203G & Syn & 2 & $\mathrm{PPCa}$ & [39], [44] \\
\hline 4522 & ND2 & $\mathrm{T}>\mathrm{C}$ & L18P & NS-PD & 2 & $\mathrm{PPCa}$ & [43], [44] \\
\hline 4561 & ND2 & $\mathrm{C}>\mathrm{T}, \mathrm{T}>\mathrm{C}$ & $\mathrm{V} 31 \mathrm{~A}, \mathrm{~A} 31 \mathrm{~V}$ & NS-Benign & 2 & PPCa, STM & [40], [41] \\
\hline 4917 & ND2 & $\mathrm{A}>\mathrm{G}, \mathrm{UN}$ & N150D, UN & NS-Benign, UN & 2 & $\mathrm{PPCa}$ & [42], [39] \\
\hline 5043 & ND2 & $\mathrm{G}>\mathrm{A}$ & A192T & NS-Benign & 2 & $\mathrm{PPCa}$ & [39] \\
\hline 5590 & tRNA-Ala & $\mathrm{G}>\mathrm{A}$ & $\mathrm{NC}$ & tRNA & 2 & $\mathrm{PPCa}$ & {$[40]$} \\
\hline 5894 & NC5 & $\mathrm{A}>+\mathrm{C}, \mathrm{A}>-\mathrm{C}$ & $\mathrm{NC}$ & $\mathrm{NC}$ & 2 & $\mathrm{PPCa}$ & {$[40]$} \\
\hline
\end{tabular}




\begin{tabular}{|c|c|c|c|c|c|c|c|}
\hline 6384 & $\operatorname{COX} 1$ & $\mathrm{G}>\mathrm{A}$ & A161T & NS-PD & 2 & $\mathrm{PPCa}$ & [43], [37] \\
\hline 6930 & COX1 & $\mathrm{G}>\mathrm{A}$ & G343X & Stopgain & 2 & $\mathrm{PPCa}$ & {$[40],[44]$} \\
\hline 7028 & COX1 & $\mathrm{C}>\mathrm{T}$ & $\mathrm{A} 375 \mathrm{~A}$ & Syn & 2 & $\mathrm{PPCa}, \mathrm{BM}, \mathrm{STM}$ & {$[41]$} \\
\hline 7293 & $\operatorname{COX} 1$ & $\mathrm{G}>\mathrm{A}$ & A464T & NS-PD & 2 & $\mathrm{PPCa}$ & [40], [37] \\
\hline 7854 & $\mathrm{COX} 2$ & $\mathrm{~T}>\mathrm{C}$ & V90A & NS-Benign & 2 & $\mathrm{PPCa}$ & {$[40],[45]$} \\
\hline 8269 & $\mathrm{COX} 2$ & $\mathrm{G}>\mathrm{G}, \mathrm{G}>\mathrm{A}$ & $\mathrm{X} 228 \mathrm{X}$ & Syn & 2 & $\mathrm{PPCa}$ & [43], [44] \\
\hline 8697 & ATP6 & $\mathrm{G}>\mathrm{A}, \mathrm{A}>\mathrm{G}$ & M57M & Syn & 2 & $\mathrm{PPCa}, \mathrm{BM}$ & [39], [41] \\
\hline 9477 & $\mathrm{COX} 3$ & $\mathrm{G}>\mathrm{C}, \mathrm{UN}$ & V91L, UN & NS-Benign, UN & 2 & PPCa, STM & [42], [41] \\
\hline 10756 & ND4L & $\mathrm{T}>\mathrm{C}$ & L96P & NS-PD & 2 & $\mathrm{PPCa}$ & {$[37]$} \\
\hline 11246 & ND4 & $\mathrm{G}>\mathrm{A}$ & A163T & NS-PD & 2 & $\mathrm{PPCa}$ & [39], [40] \\
\hline 11351 & ND4 & $\mathrm{G}>\mathrm{A}$ & A198T & NS-PD & 2 & $\mathrm{PPCa}, \mathrm{STM}$ & [37], [41] \\
\hline 11775 & ND4 & $\mathrm{G}>\mathrm{A}$ & S339N & NS-PD & 2 & $\mathrm{PPCa}$ & {$[40],[45]$} \\
\hline 11907 & ND4 & $\mathrm{T}>\mathrm{C}, \mathrm{UN}$ & V383A, UN & NS-Benign, UN & 2 & $\mathrm{PPCa}$ & {$[42],[40]$} \\
\hline 12308 & tRNA-Leu2 & $\mathrm{A}>\mathrm{G}$ & $\mathrm{NC}$ & $\mathrm{NC}$ & 2 & $\mathrm{PPCa}, \mathrm{STM}$ & [90], [41] \\
\hline 12417 & ND5 & $\mathrm{C}>-\mathrm{A}, \mathrm{C}>+\mathrm{A}$ & L20P & Frameshift & 2 & $\mathrm{PPCa}$ & {$[40]$} \\
\hline 12457 & ND5 & $\mathrm{G}>\mathrm{A}$ & $\mathrm{A} 41 \mathrm{~T}$ & NS-PD & 2 & $\mathrm{PPCa}$ & [37], [44] \\
\hline 13367 & ND5 & $\mathrm{G}>\mathrm{A}$ & G344E & NS-PD & 2 & $\mathrm{PPCa}$ & {$[40],[45]$} \\
\hline 13368 & ND5 & $\mathrm{G}>\mathrm{A}, \mathrm{A}>\mathrm{G}$ & G344G & Syn & 2 & PPCa, BM & [39], [41] \\
\hline 14560 & ND6 & $\mathrm{A}>\mathrm{A}, \mathrm{G}>\mathrm{A}$ & V38V & Syn & 2 & $\mathrm{PPCa}$ & [37], [44] \\
\hline 15375 & CYTB & $\mathrm{G}>\mathrm{A}$ & $\mathrm{G} 210 \mathrm{E}$ & NS-PD & 2 & $\mathrm{PPCa}$ & [40], [37] \\
\hline 15500 & CYTB & $\mathrm{G}>\mathrm{A}$ & $\mathrm{D} 252 \mathrm{~N}$ & NS-PD & 2 & $\mathrm{PPCa}$ & [40], [37] \\
\hline 15607 & CYTB & $\mathrm{A}>\mathrm{G}, \mathrm{G}>\mathrm{A}$ & K287K & Syn & 2 & BM, STM & {$[41]$} \\
\hline 16034 & D-Loop & $\mathrm{G}>\mathrm{A}$ & $\mathrm{NC}$ & $\mathrm{NC}$ & 2 & PPCa & {$[40]$} \\
\hline 16035 & D-Loop & $\mathrm{G}>\mathrm{A}$ & $\mathrm{NC}$ & $\mathrm{NC}$ & 2 & $\mathrm{PPCa}$ & [37], [44] \\
\hline $16182^{*}$ & D-Loop & $\mathrm{A}>\mathrm{C}$ & $\mathrm{NC}$ & $\mathrm{NC}$ & 2 & $\mathrm{PPCa}$ & {$[105]$} \\
\hline 16224 & D-Loop & $\mathrm{C}>\mathrm{C}, \mathrm{C}>\mathrm{T}$ & $\mathrm{NC}$ & $\mathrm{NC}$ & 2 & PPCa, STM & [41], [44] \\
\hline 16290 & D-Loop & $\mathrm{T}>\mathrm{T}, \mathrm{C}>\mathrm{T}$ & $\mathrm{NC}$ & $\mathrm{NC}$ & 2 & $\mathrm{PPCa}$ & [37], [44] \\
\hline 16298 & D-Loop & $\mathrm{T}>\mathrm{C}$ & $\mathrm{NC}$ & $\mathrm{NC}$ & 2 & $\mathrm{PPCa}$ & {$[105]$} \\
\hline 16311 & D-Loop & $\mathrm{C}>\mathrm{T}, \mathrm{T}>\mathrm{C}$ & $\mathrm{NC}$ & $\mathrm{NC}$ & 2 & PPCa, STM & {$[105],[41]$} \\
\hline 16320 & D-Loop & $\mathrm{C}>\mathrm{T}$ & $\mathrm{NC}$ & $\mathrm{NC}$ & 2 & PPCa, STM & [40], [41] \\
\hline 16327 & D-Loop & $\mathrm{C}>\mathrm{T}$ & $\mathrm{NC}$ & $\mathrm{NC}$ & 2 & PPCa & [40], [37] \\
\hline 16390 & D-Loop & $\mathrm{G}>\mathrm{A}$ & $\mathrm{NC}$ & $\mathrm{NC}$ & 2 & $\mathrm{PPCa}$ & [37] \\
\hline
\end{tabular}

Abbreviations: Pat, patients; UN, Unknown; NC, noncoding; Syn, synonymous; NS, non-synonymous; PD, probably damaging; PPCa, primary prostate cancer; BM, bone metastasis; STM, soft tissue metastasis. Notes: 'UN (unknown) alleles, as Parr et al. 2006 [42] reported only the mutated nucleotide position, not the allele change, thus amino acid change and prediction could not be generated. ${ }^{2}$ Pathogenicity is represented in bold and defined as a stop gain, frameshift, or is predicted as possibly damaging using PolyPhen2 [47]. *Known highly polymorphic mtDNA nucleotides involving a polyC at position 303-315, $\mathrm{A} / \mathrm{C}$ changes at positions 16182 and 16183 , and a C/T changes at position 16519 , although reported, should likely not be considered as somatic.

interpretation of pathogenicity, of the 58 somatic mtDNA mutations reported in each of two patients, 15 are predicted by PolyPhen 2 to have pathogenic potential. Of interest, two cause premature termination of transcription of ND1 (3664 $\mathrm{G}>\mathrm{A}$ ) and COl (6930 G > A) genes, and one a frameshift within ND5 at nucleotide 12417 . Reported in primary $\mathrm{PCa}$ tissues, the ND1 and ND5 variants appearing to be specific to $\mathrm{PCa}$. The $\mathrm{CO} 16930 \mathrm{G}>\mathrm{A}$ variant, which results in loss of the last 170 amino acids of the $\mathrm{C}$-terminal region of the COX1 protein (G343X), has been shown to disrupt the functional structure of Complex IV, causing multisystem mitochondrial disorder when inherited [50]. The other 12 somatic non-synonymous mutations predicted to have pathogenic potential impact the following genes: ND4 (3), CO1 (2), CYTB (2), ND5 (2), ND1 (1), ND2 (1) and ND4L (1), with all reported in primary PCa and one ND4 $11351 \mathrm{G}$ $>$ A also within a single soft tissue metastasis.

In contrast to primary $\mathrm{PCa}$, acquired mutational hotspots or recurrence of mtDNA has been suggested for metastatic PCa. Specifically, the 10398 A > G (T114A) within ND3 was reported in 7 of 10 bone metastatic tumors from a single study [41]. This variant was absent in patient matched soft tissue metastasis (lymph node, liver or lung) and primary $\mathrm{PCa}$, while observed in 3 of 21 thyroid carcinomas [51]. In contrast, a low degree of mtDNA 10398G heteroplasmy, or high degree of 10398A heteroplasmy, has been suggestive as a marker for poor prognosis in patients with non-small cell lung cancer $[52,53]$ and cervical cancer [54], respectively. The 10398 A $>$ G variant was somatically absent in 395 primary PCa's (Figure 1B), absent in a targeted study of the mtDNA ND3 gene in 77 Mexican-Mestizo men with aggressive primary $\mathrm{PCa}$ [55], and absent in the single neuroendocrine and the nine bone metastases from six PCa patients reported in this review (Table 2) [46]. While the impact of acquiring the $\mathrm{G}$-allele on PCa metastasis requires further confirmation, 10398 is a common haplogroup defining polymorphic site. Specifically, $10398 \mathrm{G}>\mathrm{A}$ variant is one of only five known polymorphic markers identifying one of the two major mitochondrial haplogroups that emerged 'out-of- 
Africa' roughly 65,000 years ago. While the ancestral within Africa L3 haplogroup and the out-of-Africa sister haplogroup $\mathrm{M}$ is defined by 10398 G-allele, the globally dispersed haplogroup $\mathrm{N}$ is defined by 10398 A-allele, with the $\mathrm{K} 1$ and J1 branches derived from a known $10398 \mathrm{~A}>\mathrm{G}$ back mutation [49]. Less common within Africa, the 10398 A-allele has been significantly associated with increased risk for invasive breast cancer in African-American woman (654 cases, 605 controls), yet this significance was absent in White Americans (879 cases, 760 controls) [56]. Additionally, in an African-American derived breast cancer cell model, the mtDNA $10398 \mathrm{G}>\mathrm{A}$ variant was shown to increase ROS production, confer resistance to apoptosis and promoted metastasis [57]. As with the somatic $10398 \mathrm{~A}>\mathrm{G}$ variant, the acquired ND4 $11719 \mathrm{G}>\mathrm{A}$ variant reported in four of 10 metastatic [41] and a single primary $\mathrm{PCa}$ [39], is a known polymorphic back mutation defining the $\mathrm{H} 5 \mathrm{a} 1 \mathrm{~b}$ and H27 European-predominant haplogroups from the original R0 (previously pre-HV haplogroup) defining 11719 A $>$ G polymorphism. The predominance of haplogroup defining polymorphic sites to acquire somatic mtDNA mutations during metastases needs further exploration.

It is well established that mutagenic mechanisms exhibit different mutational DNA signatures. For mtDNA, it has been speculated that mutations would arise from the proximity to ROS species generated by OXPHOS [55]. ROS-induced mutations are characterized by guanine oxidation resulting in an over representation of $\mathrm{G}: \mathrm{C}>$ $\mathrm{T}: \mathrm{A}$ transversion mutations [58]. However, mutations identified in mtDNA in $\mathrm{PCa}$ predominantly involve $\mathrm{C}>\mathrm{T}(57 \%)$ and $\mathrm{T}>\mathrm{C}(35.9 \%)$ transitions, with only 1.3\% G:C > T:A mutations observed. This is comparable to mtDNA mutations in all 1,675 tumor types sequenced as part of the TCGA effort [40], with speculated ROSassociated mutations accounting only for $4 \%$ of variants. Furthermore, Ju et al., 2014, found a strong bias of C $>$ T and $\mathrm{A}>\mathrm{G}$ mutations on the mtDNA heavy strand and $\mathrm{G}>\mathrm{A}$ and $\mathrm{T}>\mathrm{C}$ bias on the light strand in cancer, which holds true for PCa.

Besides SNVs and indels, large mtDNA deletions have been reportedly acquired during prostate tumorigenesis. Using long-range amplification and Southern blot analysis, a 2001 study observed large somatic mtDNA deletions in all 34 primary PCa specimens [59]. Furthermore, they suggest that the number of large deletions observed increases proportionally with the patient's age. While men in their forties reportedly present with on average 1.25 additional deletions, this increases to 5.9 additional deletions in men in their seventies, including a common $14.8 \mathrm{~Kb}$ deletion observed in all men over 60 years. A single group has also reported the acquisition of a common 3,379 basepair mtDNA deletion in $\mathrm{PCa}$ [60]. This variant, which knocks out the terminal end of $N D 4 L$, the complete ND4, most of the ND5 genes, and three tRNAs, was further reported to predict the presence of malignant tumor tissue proximal to biopsies appearing histologically normal [61]. The significance of these large deletions requires further verification.

\section{BIOLOGICAL IMPACT OF PCAASSOCIATED MITOCHONDRIAL MUTATIONS}

Compared with the nuclear genome, the mitochondrial genome is largely coding. It is well established that mutations in mtDNA contribute to mitochondrial dysfunction as shown in mitochondrial diseases, cell models and cancer [1, 5, 7]. Mitochondrial dysfunction has been implicated in tumor growth, metastatic potential and therapeutic response rate in several cancers [9]. The inherited mtDNA $6124 \mathrm{~T}>\mathrm{C}$ mutation in the $\mathrm{CO} 1$ gene has been shown to alter reactive oxygen and nitrogen species and proliferation in a patient with PCa by using patient derived cybrid models (a fusion of enucleated patient fibroblasts to $14 \mathrm{~B}$ rho cell not containing mitochondrial DNA) [62], implicating an effect of variants in the mtDNA on tumorigenesis. Using the same technique, they introduced this mutation in PC3 PCa cells and identified a resistance to the apoptotic effects of statins [63]. Metastatic potential was shown to be altered in PCa cells in the bone microenvironment in cybrid models by inheriting an mtDNA $8993 \mathrm{~T}>\mathrm{G}$ mutation in the ATP6 gene [64]. Cybrids allow researchers to introduce mutated mtDNA into a prostate epithelial or cancer cell line and investigate if the mutations change cellular characteristics [65]. These modified cells can be injected into mice and, as shown for lung cancer cells, tumors can acquire metastatic potential from mtDNA replacement from a highly metastatic cell line [66]. These techniques are becoming more reliable and have been implicated in restoring mutated mtDNA in patients carrying inherited mitochondrial disease, with further advances opening the possibility to restore these mutations in cancer patients [67].

Although ROS-induced mtDNA mutations appear to be rare in $\mathrm{PCa}$, based on mutational signatures, both the inherited $6142 \mathrm{~T}>\mathrm{C}$ and $8993 \mathrm{~T}>\mathrm{G}$ PCa associated mtDNA mutations have been shown to be ROS-producing [68]. Notably, elevated levels of ROS are evident in numerous cancer types, including $\mathrm{PCa}$ [69]. A recent review addresses the functions, and potential mechanisms, of elevated ROS-production in both promoting and suppressing tumor development [70]. Specifically, increased ROS-production has been associated with uncontrolled cell cycle, clonogenicity, invasion and metastasis in PCa cells [71]. Further functional support comes from a study showing that ROS-producing mtDNA mutations play a role in regulating lung-derived tumor cell metastasis, with potential for therapeutic intervention [66]. We have identified 80 recurrent (presenting in two or more patients) somatic PCa associated mtDNA mutations reported to date, with roughly $19 \%$ having a predicted 
impact on protein function. The impact of mtDNA mutations acquired during prostate tumorigenesis on ROSproduction, however, remains unknown. Interestingly, Arnold et al., (2009) suggests that OXPHOS complex IV mutations increase ROS levels (data not shown) [64], while in this review we observe an over representation of somatic mutations within the coding genes of this highly conserved complex. The significance of somatic mtDNA mutations driving ROS-production and in turn prostate tumorigenesis, requires further investigation.

\section{MITOCHONDRIAL DNA COPY NUMBER ALTERATIONS IN PCA}

Mitochondrial copy number in normal cells is regulated by ATP demand and hypoxia [72]. Although there is variability in the number of copies of mtDNA in a cell, the mtDNA copy number tends to be heritable, declining with age $[73,74]$. mtDNA copy number has been studied in blood and cancer tissue of patients. A metaanalysis of peripheral blood mtDNA copy number in 36 cancer studies found no significant correlation of mtDNA copy number and cancer risk in general. Subgroup analysis showed a positive correlation for risk of lymphoma and breast cancer $(\mathrm{OR}=1.645,95 \% \mathrm{CI}=1.117-2.421$, $P=0.012 ; \mathrm{OR}=1.721,95 \% \mathrm{CI}=1.130-2.622, P=0.011)$ [75]. A meta-analysis investigating cancer prognosis included both blood $(n=6)$ and tissue $(n=14)$ studies and established that a high mtDNA copy number in blood was associated with reduced disease-free survival $(\mathrm{HR}=1.582,95 \% \mathrm{CI}: 1.026-2.439, p=0.038)$, and a high mtDNA copy number in tissue in contrast was associated with an increased disease free survival $(\mathrm{HR}=0.593,95 \%$ CI: $0.411-0.857, p=0.005)$ [76]. A study investigating urological cancers, including $\mathrm{PCa}$ reported an increase in cell free circulating mtDNA in cancer patients [77]. In PCa specific studies, a single retrospective study, although underpowered (193 cases and 194 controls), reported an association between mtDNA copy number in peripheral blood leucocytes and PCa risk [78]. However, this finding was not replicated in a larger prospective nested study (793 cases and 790 controls) [79]. Inverse associations were also found between mtDNA copy number and aggressive and non-aggressive $\mathrm{PCa}$, respectively, which may be a direct result of study design, with the authors calling for independent replication. A study investigating 1751 nonHispanic white men found that low mtDNA copy number in blood corresponded to a higher risk disease ORs of 1.33 (95\% CI, 0.89-1.98; $p=0.17$ ) and 1.53 (95\% CI, $1.02-2.30 ; p=0.04)$, for second and first tertiles respectively and increased risk of disease progression (HR, 1.56; 95\% CI, 0.96-2.54; $p=0.07$ ) [80].

There is less information available on tissue-derived mtDNA copy number, with one small study reporting a higher mtDNA content in PCa versus normal prostate cells in seven of nine cases [81]. A reduction in mtDNA content in normal, BPH and PCa tissue was reported for American men of African versus European ancestry and in men aged $\geq 60$ years [82]. A recent study analyzed TCGA cancer genome data for mtDNA copy number across 22 cancers compared to normal tissue. It observed a trend for several cancers, particularly bladder, breast and kidney, to be depleted of mtDNA and found an association with somatic alteration in the nuclear genome and respiratory gene expression, suggesting that mitochondrial activity was suppressed in these cancers [83]. In contrast and uniquely to $\mathrm{PCa}$, the same study reported an inverse correlation between mtDNA copy number and mitochondrial related nuclear gene expression. The mechanism through which these changes in mitochondrial copy number occur are unclear.

\section{MITOCHONDRIAL GENOME VARIANCE IN CLINCIAL PRACTICE}

Although PCa is the most commonly diagnosed male cancer and has the second highest cancer mortality rate in men living in the most economically developed countries [84], clinical management is complicated by extreme variability in disease course. This includes symptom-free indolent to lethal disease, and a wide range of treatment options, including active surveillance, radiotherapy, hormone therapy, radical prostatectomy, as well as combination therapies. While PCa treatment has a high curability rate [85], radiotherapy and surgery have notable side effects, including erectile dysfunction, urinary incontinence and pain [86], and hormone treatment is associated with decreased libido, obesity and osteoporosis [87]. In addition to digital rectal examination, a suspected diagnosis is reliant on blood-based prostate specific antigen (PSA) testing which, since its introduction in the mid 1990's, has greatly increased the incidence of PCa with minimal impact on morbidity and mortality [88]. Actual PCa diagnosis can only be made with a prostate biopsy and associated pathological Gleason grading [89]. New non-invasive biomarkers are therefore urgently required to improve clinical management of $\mathrm{PCa}$.

The first acquired mtDNA variant to be implicated in PCa and developed as a commercial biomarker for suggestive false negative biopsy identification is based on the previously described large $3.4 \mathrm{~Kb}$ deletion [60]. While this biomarker was implicated in men of European ancestry, it was suggested to be absent in an Australian [44] and an African study [37]. Other suggested mtDNAbased biomarkers requiring further clarification include an elevated level of acquired tRNAs mutations and an increased level of heteroplasmy [39, 43]. Correcting for regional length, we found tRNAs Pro and Ala to be most frequently mutated when considering all whole mitochondrial genome PCa studies. While a trend towards tRNA somatic mutations has been reported in European studies, the single African study reports fewer somatic 
tRNA mutations than expected [37]. The observation of increased frequency of homoplasmic variation in metastatic over primary PCa when considering all PCa associated mutations and therefore potential as oncogenic drivers, calls for further studies focused on metastatic disease.

More recently, a correlation between total number of somatic mtDNA variants (defined as mutational load) and Gleason score at diagnosis has been suggested from an African-based [37] and Australianbased study [44]. Furthermore, the latter reported a correlation between a higher mutational load and risk of biochemical relapse after surgery, with a Hazard Ratio (HR) of 2.17 for $\geq 1$ SNVs and a HR of 3.82 for $\geq 2$ SNV. Both studies noted a lack of recurrent mutations. These studies suggest that rather than a single mtDNA mutation, the total mtDNA mutational burden is a potential biomarker of aggressive disease. Combining the four studies where data was available to associate number of somatic mtDNA mutations with clinical characteristics [37, 39, 43, 44], we show a significant increase in the number of variants with sample combined Gleason score ( $p=<0.0001)$ (Figure 2A), with PSA level before radical prostatectomy or at diagnosis $(p=0.30)$ (Figure 2B) and age of patient at radical prostatectomy or diagnosis ( $p=<0.0001)$ (Figure 2C), further supporting the proposal that mtDNA mutation burden may be an indicator of PCa. The McCrow et al., 2016 paper further suggests that these mutations may not be causal but rather an indicator of a higher degree of mutagenesis, while also suggesting an upper limitation of cellular tolerance for mutational load [37]. Although less frequent, somatic mtDNA variants have also been identified in tumor-matched urine and plasma from PCa patients [90]. Two other studies revealed circulating cell free tumor mtDNA in PCa and further showed a correlation with survival or early PSA recurrence [91, 92].

The mitochondrial genome has clear advantages over the nuclear genome as a target for PCa biomarker development, including its significantly smaller genome size, increased biological stability and abundance, as well as providing a logical correlation between observed clinical heterogeneity and the uniquely mitochondrial phenomenon of heteroplasmy. As formalin fixation and paraffin embedding (FFPE) of prostate tissue is routine practice during pathological diagnosis, the process itself increases potential for the introduction of mutations and DNA breaks and in turn sequencing error [93]. These FFPE-derived artifacts are less prominent in mtDNA than nuclear DNA due to the higher stability and abundance. The small size and abundance also facilitates the detection of small quantities of cell free mtDNA or circulating tumor cells in diagnostically appropriate bodily fluids such as blood, urine or semen. Additionally, whole exome or genome sequencing efforts often overlook the mitochondrial genome, even though higher sequencing coverage is inadvertently generated relative to the targeted nuclear genome. Advances in analytical software to address heteroplasmy will greatly advance the utilization of the mtDNA data, which is essentially generated as a byproduct of WGS/WES. Although RNA sequencing allows for variant calling of the expressed mtDNA genes,
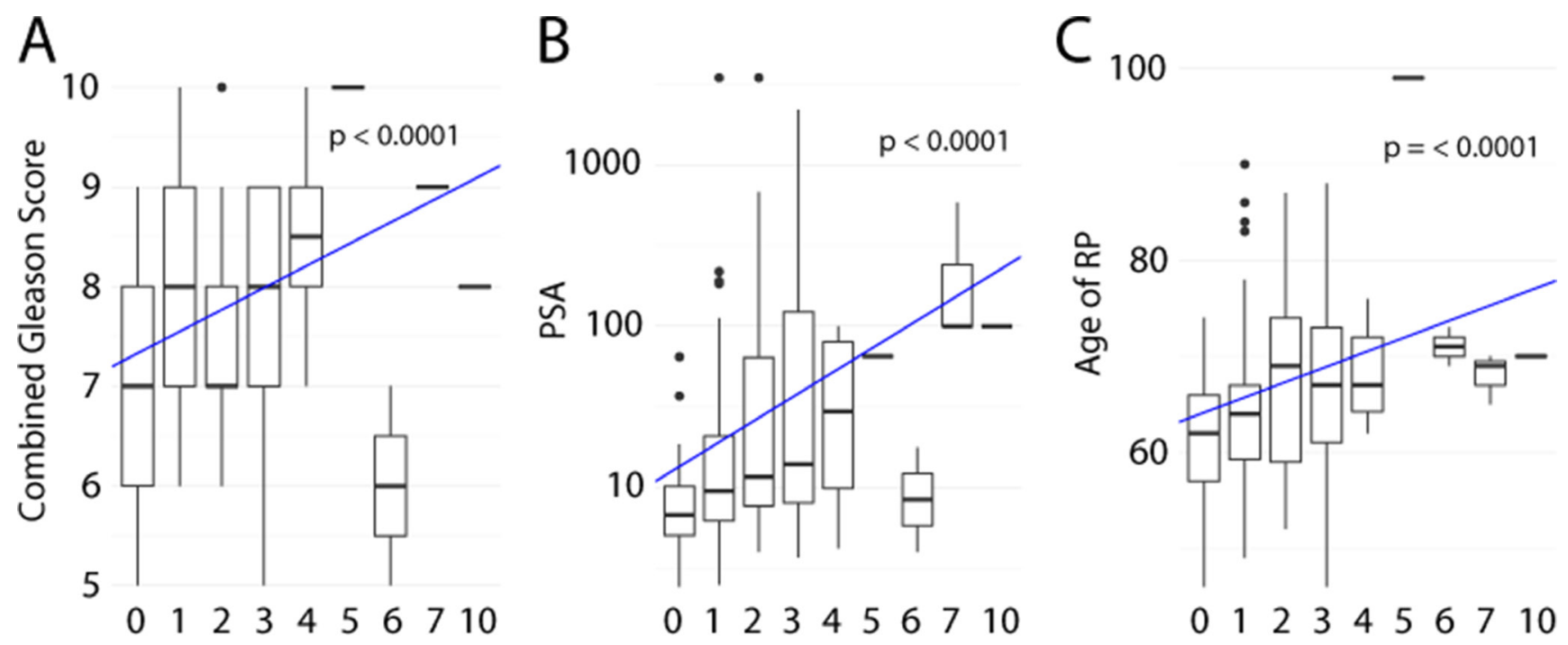

\section{Number of Variants}

Figure 2: Number of somatic mtDNA variants correlated with clinical characteristics from four studies. (A) Sample combined Gleason score. (B) PSA level before radical prostatectomy or at diagnosis. (C) Age of patient at radical prostatectomy or diagnosis. The line and $p$-value represent the linear model for given characteristic by mutation number, generated using $\mathrm{R}$ with ggplot2 package $[97,98]$. 
one should be cautious that this does not cover the whole mtDNA and introduces coverage bias through differential RNA half live and potential sequence bias by post transcriptional modifications of RNA [94].

\section{CONCLUDING REMARKS}

In addition to the nuclear genome, the mitochondrial genome not only carries potential to increase a man's risk for PCa via germline variation, but the maternally inherited genome may also acquire mutations during tumorigenesis. While no single population-specific nonAfrican haplogroup appears to definitively increase PCa risk, further studies would be beneficial to address relevance and potential aggressive disease association in African populations. Furthermore, the observation that inherited mtDNA mutations provides a direct biological impact on mitochondrial function and $\mathrm{PCa}$ tumorigenesis, calls for further investigation of the role of germline mtDNA variation in $\mathrm{PCa}$.

While a maximum of seven acquired mtDNA mutations were reported in primary $\mathrm{PCa}$ from nonAfricans, up to ten mutations were found within a single African study biased towards aggressive disease pathology at presentation. Studies focused on metastatic PCa are limited. A 2015 study and unpublished mtDNA data presented in this review, suggest mutational burden, oncogenic potential, level of heteroplasmy, and recurrence rate for somatic mtDNA mutations increase with metastasis. Overall there is a paucity of evidence for ROS-induced mutagenesis, while meta-analysis for whole mitochondrial genome data shows preferential acquisition of variants in genes coding for the OXPHOS complex IV (cytochrome $c$ oxidase, COX), as well as specific regulators (tRNA-Pro, tRNA-Ala and RNR2) and the control region (D-loop). The pathogenic significance for the abundance of somatic mtDNA mutations in the highly conserved $\mathrm{COX}$ coding genes ( $\mathrm{CO} 1, \mathrm{CO} 2$, and CO3), which form the core of the complex IV enzyme in which the redox reaction takes place, calls for further investigation. This is further supported by known pathogenetic mutations within this region, either directly associated with mitochondrial diseases [95], or biological impact via altering levels of ROS production [62]. Apart from SNVs and indels, mtDNA large deletions and copy number variants have also been reported to be associated with $\mathrm{PCa}$, although the significance of these variants requires further investigation.

Although still in early stages of discovery for PCa, the significance of the mitochondrial genome to influence cancer progression and cancer therapy is gaining rapid momentum [7]. In particular, the role of mtDNA mutations to induce metastasis via elevating ROS production, provides exciting opportunities for therapeutic intervention via inhibiting ROS signaling [96]. We argue that the hope for preventing and treating clinically heterogeneous $\mathrm{PCa}$ lies in the broad application of individualized genomics, which forms the basis of precision medicine. This review provides evidence that supports the inclusion of the mitochondrial genome in the future of $\mathrm{PCa}$ precision medicine. The mitochondrial genome provides not only an explanation for extreme PCa clinical heterogeneity via co-expression of inherited and somatic mtDNA variation in varying ratios (heteroplasmy), but has benefits for diagnostic and/or prognostic biomarker development, as well as improving therapeutic intervention.

\section{Abbreviations}

mtDNA, mitochondrial DNA; PCa, prostate cancer; OXPHOS, oxidative phosphorylation; GWAS, genome wide association study; TCGA, the cancer genome atlas; SCNAs, somatic copy number alterations; ROS, reactive oxygen species; D-loop, displacement loop; SNV, single nucleotide variant; ICGC, International Cancer Genome Consortium; rCRS, revised Cambridge Reference Sequence; PSA, prostate specific antigen; HR, hazard ratio; FFPE, formalin fixation and paraffin embedding.

\section{Author contributions}

All authors contributed to the writing of this article. Additionally, A.M.F.K. and V.M.H. contributed to the concept and design, A.M.F.K. performed meta-analyses, N.M.C. and C.M.H. provided unpublished metastatic prostate cancer data, while E.K.F.C. provided the bioinformatics and biostatistics support for unpublished data.

\section{CONFLICTS OF INTEREST}

The authors declare no conflicts of interest.

\section{FUNDING}

A.M.F.K. is supported by the Australian Prostate Cancer Research Centre (APCRC) New South Wales and N.M.C. and C.M.H. by the APCRC-Epworth. E.K.F.C. is supported by the Movember Australia and the Prostate Cancer Foundation Australia (PCFA) funded Movember Revolutionary Team Award (MRTA) to the Garvan Institute of Medical Research ProMis program and V.M.H. is supported by the University of Sydney Foundation and Petre Foundation, Australia.

\section{REFERENCES}

1. Kim A. Mitochondrial DNA somatic mutation in cancer. Toxicol Res. 2014; 30:235-242.

2. Ngo DC, Ververis K, Tortorella SM, Karagiannis TC. Introduction to the molecular basis of cancer metabolism and the Warburg effect. Mol Biol Rep. 2015; 42:819-823. 
3. Zhang S, Yang C, Yang Z, Zhang D, Ma X, Mills G, Liu Z. Homeostasis of redox status derived from glucose metabolic pathway could be the key to understanding the Warburg effect. Am J Cancer Res. 2015; 5:928-944.

4. Zong WX, Rabinowitz JD, White E. Mitochondria and Cancer. Mol Cell. 2016; 61:667-676.

5. Wallace DC. Mitochondria and cancer. Nat Rev Cancer. 2012; 12:685-698.

6. Vyas S, Zaganjor E, Haigis MC. Mitochondria and Cancer. Cell. 2016; 166:555-566.

7. Van Gisbergen MW, Voets AM, Starmans MHW, de Coo IFM, Yadak R, Hoffmann RF, Boutros PC, Smeets HJM, Dubois L, Lambin P. How do changes in the mtDNA and mitochondrial dysfunction influence cancer and cancer therapy? Challenges, opportunities and models. Mutation research Reviews in mutation research. 2015; 764:16-30.

8. Boström PJ, Bjartell AS, Catto JWF, Eggener SE, Lilja H, Loeb S, Schalken J, Schlomm T, Cooperberg MR. Genomic Predictors of Outcome in Prostate Cancer. Eur Urol. 2015; 68:1033-1044.

9. Giampazolias E, Tait SWG. Mitochondria and the hallmarks of cancer. FEBS J. 2015.

10. Peisch SF, Van Blarigan EL, Chan JM, Stampfer MJ, Kenfield SA. Prostate cancer progression and mortality: a review of diet and lifestyle factors. World J Urol. 2017; 35:867-874.

11. Mucci LA, Hjelmborg JB, Harris JR, Czene K, Havelick DJ, Scheike T, Graff RE, Holst K, Möller S, Unger RH, McIntosh C, Nuttall E, Brandt I, et al. Familial Risk and Heritability of Cancer Among Twins in Nordic Countries. JAMA. 2016; 315:68-76.

12. Kiciński M, Vangronsveld J, Nawrot TS. An epidemiological reappraisal of the familial aggregation of prostate cancer: a meta-analysis. PLoS One. 2011; 6:e27130.

13. DeSantis CE, Siegel RL, Sauer AG, Miller KD, Fedewa SA, Alcaraz KI, Jemal A. Cancer statistics for African Americans, 2016: Progress and opportunities in reducing racial disparities. CA Cancer J Clin. 2016; 66:290-308.

14. Chen R, Ren S, Sun Y. Genome-wide association studies on prostate cancer: the end or the beginning? Protein Cell. 2013.

15. Amin Al Olama A, Kote-Jarai Z, Schumacher FR, Wiklund F, Berndt SI, Benlloch S, Giles GG, Severi G, Neal DE, Hamdy FC, Donovan JL, Hunter DJ, Henderson BE, et al. A meta-analysis of genome-wide association studies to identify prostate cancer susceptibility loci associated with aggressive and non-aggressive disease. Hum Mol Genet. $2013 ; 22: 408-415$.

16. Srinivasan S, Clements JA, Batra J. Single nucleotide polymorphisms in clinics: Fantasy or reality for cancer? Crit Rev Clin Lab Sci. 2016; 53:29-39.

17. Amin Al Olama A, Benlloch S, Antoniou AC, Giles GG, Severi G, Neal DE, Hamdy FC, Donovan JL, Muir K, Schleutker J, Henderson BE, Haiman CA, Schumacher FR, et al. Risk Analysis of Prostate Cancer in PRACTICAL, a Multinational Consortium, Using 25 Known Prostate Cancer Susceptibility Loci. Cancer Epidemiol Biomarkers Prev. 2015; 24:1121-1129.

18. Lynch HT, Kosoko-Lasaki O, Leslie SW, Rendell M, Shaw T, Snyder C, D'Amico AV, Buxbaum S, Isaacs WB, Loeb S, Moul JW, Powell I. Screening for familial and hereditary prostate cancer. Int J Cancer. 2016; 138:2579-2591.

19. Cancer Genome Atlas Research Network. The molecular taxonomy of primary prostate cancer. Cell. 2015; 163:10111025.

20. Valdés-Mora F, Clark SJ. Prostate cancer epigenetic biomarkers: next-generation technologies. Oncogene. 2015; 34:1609-1618.

21. Baca SC, Prandi D, Lawrence MS, Mosquera JM, RomanelA, Drier Y, Park K, Kitabayashi N, MacDonald TY, Ghandi M, Van Allen E, Kryukov GV, Sboner A, et al. Punctuated evolution of prostate cancer genomes. Cell. 2013; 153:666677.

22. Bosland MC, Ozten N, Eskra JN, Mahmoud AM. A Perspective on Prostate Carcinogenesis and Chemoprevention. Current pharmacology reports. 2015; 1:258-265.

23. Penta JS, Johnson FM, Wachsman JT, Copeland WC. Mitochondrial DNA in human malignancy. Mutation Research/Reviews in Mutation Research. 2001; 488:119-133.

24. Boulet L, Karpati G, Shoubridge EA. Distribution and threshold expression of the tRNA(Lys) mutation in skeletal muscle of patients with myoclonic epilepsy and ragged-red fibers (MERRF). Am J Hum Genet. 1992; 51:1187-1200.

25. King MP, Attardi G. Human cells lacking mtDNA: repopulation with exogenous mitochondria by complementation. Science 1989; 246:500-503.

26. Park JS, Sharma LK, Li H, Xiang R, Holstein D, Wu J, Lechleiter J, Naylor SL, Deng JJ, Lu J, Bai Y. A heteroplasmic, not homoplasmic, mitochondrial DNA mutation promotes tumorigenesis via alteration in reactive oxygen species generation and apoptosis. Hum Mol Genet. 2009; 18:1578-1589.

27. Bratt O, Drevin L, Akre O, Garmo H, Stattin P. Family History and Probability of Prostate Cancer, Differentiated by Risk Category: A Nationwide Population-Based Study. J Natl Cancer Inst. 2016; 108.

28. Kenney MC, Chwa M, Atilano SR, Falatoonzadeh P, Ramirez C, Malik D, Tarek M, Del Carpio JC, Nesburn AB, Boyer DS, Kuppermann BD, Vawter MP, Jazwinski SM, et al. Molecular and bioenergetic differences between cells with African versus European inherited mitochondrial DNA haplogroups: implications for population susceptibility to diseases. Biochim Biophys Acta. 2014; 1842:208-219.

29. DeSantis C, Naishadham D, Jemal A. Cancer statistics for African Americans, 2013. CA Cancer J Clin. 2013; 63:151166.

30. McGinley KF, Tay KJ, Moul JW. Prostate cancer in men of African origin. Nat Rev Urol. 2016; 13:99-107.

31. Booker LM, Habermacher GM, Jessie BC, Sun QC, Baumann AK, Amin M, Lim SD, Fernandez-Golarz C, 
Lyles RH, Brown MD, Marshall FF, Petros JA. North American white mitochondrial haplogroups in prostate and renal cancer. J Urol. 2006; 175:468-72.

32. Canter JA, Kallianpur AR, Fowke JH. Re: North American white mitochondrial haplogroups in prostate and renal cancer. J Urol. 2006; 176:2308-9.

33. Wang L, McDonnell SK, Hebbring SJ, Cunningham JM, St Sauver J, Cerhan JR, Isaya G, Schaid DJ, Thibodeau SN. Polymorphisms in mitochondrial genes and prostate cancer risk. Cancer Epidemiol Biomarkers Prev. 2008; 17:3558-3566.

34. Fachal L, Gómez-Caamaño A, Alvarez Iglesias V, Gómez Carballa A, Calvo P, Salas A, Vega A. No association between typical European mitochondrial variation and prostate cancer risk in a Spanish cohort. J Hum Genet. 2014; 59:411-414.

35. Giorgi EE, Li Y, Caberto CP, Beckman KB, Lum-Jones A, Haiman CA, Le Marchand L, Stram DO, Saxena R, Cheng I. No association between the mitochondrial genome and prostate cancer risk: The Multiethnic Cohort. Cancer Epidemiol Biomarkers Prev. 2016.

36. Chan EKF, Hardie RA, Petersen DC, Beeson K, Bornman RMS, Smith AB, Hayes VM. Revised timeline and distribution of the earliest diverged human maternal lineages in southern Africa. PLoS One. 2015; 10:e0121223.

37. McCrow JP, Petersen DC, Louw M, Chan EKF, Harmeyer K, Vecchiarelli S, Lyons RJ, Bornman MSR, Hayes VM. Spectrum of mitochondrial genomic variation and associated clinical presentation of prostate cancer in South African men. Prostate. 2016; 76:349-358.

38. Scott TA, Arnold R, Petros JA. Mitochondrial Cytochrome c Oxidase subunit 1 Sequence Variation in Prostate Cancer. Scientifica (Cairo). 2012; 2012:701810.

39. Lindberg J, Mills IG, Klevebring D, Liu W, Neiman M, Xu J, Wikström P, Wiklund P, Wiklund F, Egevad L, Grönberg H. The mitochondrial and autosomal mutation landscapes of prostate cancer. Eur Urol. 2013; 63:702-708.

40. Ju YS, Alexandrov LB, Gerstung M, Martincorena I, NikZainal S, Ramakrishna M, Davies HR, Papaemmanuil E, Gundem G, Shlien A, Bolli N, Behjati S, Tarpey PS, et al. Origins and functional consequences of somatic mitochondrial DNA mutations in human cancer. elife. 2014; 3.

41. KalsbeekAM, Chan EF, Grogan J, Petersen DC, Jaratlerdsiri W, Gupta R, Lyons RJ, Haynes AM, Horvath LG, Kench JG, Stricker PD, Hayes VM. Mutational load of the mitochondrial genome predicts pathological features and biochemical recurrence in prostate cancer. Aging (Albany NY). 2016; 8:2702-2712. https://doi.org/10.18632/aging.101044.

42. Parr RL, Dakubo GD, Crandall KA, Maki J, Reguly B, Aguirre A, Wittock R, Robinson K, Alexander JS, BirchMachin MA, Abdel-Malak M, Froberg MK, Diamandis EP, et al. Somatic mitochondrial DNA mutations in prostate cancer and normal appearing adjacent glands in comparison to age-matched prostate samples without malignant histology. J Mol Diagn. 2006; 8:312-319.
43. Kloss-Brandstätter A, Schäfer G, Erhart G, Hüttenhofer A, Coassin S, Seifarth C, Summerer M, Bektic J, Klocker H, Kronenberg F. Somatic mutations throughout the entire mitochondrial genome are associated with elevated PSA levels in prostate cancer patients. Am J Hum Genet. 2010; 87:802-812.

44. Kalsbeek AMF, Chan EFK, Grogan J, Petersen DC, Jaratlerdsiri W, Gupta R, Lyons RJ, Haynes AM, Horvath LG, Kench JG, Stricker PD, Hayes VM. Mutational load of the mitochondrial genome predicts pathological features and biochemical recurrence in prostate cancer. Aging. 2016.

45. International Cancer Genome Consortium, Hudson TJ, Anderson W, Artez A, Barker AD, Bell C, Bernabé RR, Bhan MK, Calvo F, Eerola I, Gerhard DS, Guttmacher A, Guyer $\mathrm{M}$, et al. International network of cancer genome projects. Nature. 2010; 464:993-998.

46. Hong MKH, Macintyre G, Wedge DC, Van Loo P, Patel K, Lunke S, Alexandrov LB, Sloggett C, Cmero M, Marass F, Tsui D, Mangiola S, Lonie A, et al. Tracking the origins and drivers of subclonal metastatic expansion in prostate cancer. Nat Commun. 2015; 6:6605.

47. Adzhubei IA, Schmidt S, Peshkin L, Ramensky VE, Gerasimova A, Bork P, Kondrashov AS, Sunyaev SR. A method and server for predicting damaging missense mutations. Nat Methods. 2010; 7:248-249.

48. Flanagan SE, Patch AM, Ellard S. Using SIFT and PolyPhen to predict loss-of-function and gain-of-function mutations. Genet Test Mol Biomarkers. 2010; 14:533-537.

49. Van Oven M, Kayser M. Updated comprehensive phylogenetic tree of global human mitochondrial DNA variation. Hum Mutat. 2009; 30:E386-94.

50. Bruno C, Martinuzzi A, Tang Y, Andreu AL, Pallotti F, Bonilla E, Shanske S, Fu J, Sue CM, Angelini C, DiMauro S, Manfredi G. A stop-codon mutation in the human mtDNA cytochrome c oxidase I gene disrupts the functional structure of complex IV. Am J Hum Genet. 1999; 65:611-620.

51. Yeh JJ, Lunetta KL, van Orsouw NJ, Moore FD, Mutter GL, Vijg J, Dahia PL, Eng C. Somatic mitochondrial DNA (mtDNA) mutations in papillary thyroid carcinomas and differential mtDNA sequence variants in cases with thyroid tumours. Oncogene. 2000; 19:2060-2066.

52. Qi Y, Wei Y, Wang Q, Xu H, Wang Y, Yao A, Yang H, Gao Y, Zhou F. Heteroplasmy of mutant mitochondrial DNA A10398G and analysis of its prognostic value in non-small cell lung cancer. Oncol Lett. 2016; 12:3081-3088.

53. Xu H, He W, Jiang HG, Zhao H, Peng XH, Wei YH, Wei JN, Xie CH, Liang C, Zhong YH, Zhang G, Deng D, Zhou YF, et al. Prognostic value of mitochondrial DNA content and G10398A polymorphism in non-small cell lung cancer. Oncol Rep. 2013; 30:3006-3012.

54. Feng D, Xu H, Li X, Wei Y, Jiang H, Xu H, Luo A, Zhou F. An association analysis between mitochondrial DNA content, G10398A polymorphism, HPV infection, and the prognosis of cervical cancer in the Chinese Han population. Tumour Biol. 2016; 37:5599-5607. 
55. Canto P, Benítez Granados J, Martínez Ramírez MA, Reyes E, Feria-Bernal G, García-García E, Tejeda ME, Zavala E, Tapia A, Rojano-Mejía D, Méndez JP. Genetic variants in ATP6 and ND3 mitochondrial genes are not associated with aggressive prostate cancer in Mexican-Mestizo men with overweight or obesity. Aging Male. 2016; 19:187-191.

56. Canter JA, Kallianpur AR, Parl FF, Millikan RC. Mitochondrial DNA G10398A polymorphism and invasive breast cancer in African-American women. Cancer Res. 2005; 65:8028-8033.

57. Kulawiec M, Owens KM, Singh KK. mtDNA G10398A variant in African-American women with breast cancer provides resistance to apoptosis and promotes metastasis in mice. J Hum Genet. 2009; 54:647-654.

58. Delaney S, Jarem DA, Volle CB, Yennie CJ. Chemical and biological consequences of oxidatively damaged guanine in DNA. Free Radic Res. 2012; 46:420-441.

59. Jessie BC, Sun CQ, Irons HR, Marshall FF, Wallace DC, Petros JA. Accumulation of mitochondrial DNA deletions in the malignant prostate of patients of different ages. Exp Gerontol. 2001; 37:169-174.

60. Maki J, Robinson K, Reguly B, Alexander J, Wittock R, Aguirre A, Diamandis EP, Escott N, Skehan A, Prowse O, Thayer RE, Froberg MK, Wilson MJ, et al. Mitochondrial genome deletion aids in the identification of false- and truenegative prostate needle core biopsy specimens. Am J Clin Pathol. 2008; 129:57-66.

61. Robinson K, Creed J, Reguly B, Powell C, Wittock R, Klein D, Maggrah A, Klotz L, Parr RL, Dakubo GD. Accurate prediction of repeat prostate biopsy outcomes by a mitochondrial DNA deletion assay. Prostate Cancer Prostatic Dis. 2010; 13:126-131.

62. Arnold RS, Sun Q, Sun CQ, Richards JC, O'Hearn S, Osunkoya AO, Wallace DC, Petros JA. An inherited heteroplasmic mutation in mitochondrial gene COI in a patient with prostate cancer alters reactive oxygen, reactive nitrogen and proliferation. BioMed research international. 2013; 2013:239257.

63. Sun Q, Arnold RS, Q Sun C, A Petros J. A mitochondrial DNA mutation influences the apoptotic effect of statins on prostate cancer. Prostate. 2015; 75:1916-1925.

64. Arnold RS, Sun CQ, Richards JC, Grigoriev G, Coleman IM, Nelson PS, Hsieh CL, Lee JK, Xu Z, Rogatko A, Osunkoya AO, Zayzafoon M, Chung L, et al. Mitochondrial DNA mutation stimulates prostate cancer growth in bone stromal environment. Prostate. 2009; 69:1-11.

65. Heller S, Schubert S, Krehan M, Schäfer I, Seibel M, Latorre D, Villani G, Seibel P. Efficient repopulation of genetically derived rho zero cells with exogenous mitochondria. PLoS One. 2013; 8:e73207.

66. Ishikawa $\mathrm{K}$, Takenaga $\mathrm{K}$, Akimoto M, Koshikawa N, Yamaguchi A, Imanishi H, Nakada K, Honma Y, Hayashi JI. ROS-generating mitochondrial DNA mutations can regulate tumor cell metastasis. Science. 2008; 320:661-664.
67. Jo A, Ham S, Lee GH, Lee YI, Kim S, Lee YS, Shin JH, Lee Y. Efficient Mitochondrial Genome Editing by CRISPR/Cas9. BioMed research international. 2015; 2015:305716.

68. Petros JA, Baumann AK, Ruiz-Pesini E, Amin MB, Sun CQ, Hall J, Lim S, Issa MM, Flanders WD, Hosseini SH, Marshall FF, Wallace DC. mtDNA mutations increase tumorigenicity in prostate cancer. Proc Natl Acad Sci USA. 2005; 102:719-724.

69. Prasad S, Gupta SC, Tyagi AK. Reactive oxygen species (ROS) and cancer: Role of antioxidative nutraceuticals. Cancer Lett. 2017; 387:95-105.

70. Galadari S, Rahman A, Pallichankandy S, Thayyullathil F. Reactive oxygen species and cancer paradox: To promote or to suppress? Free Radic Biol Med. 2017; 104:144-164.

71. Kumar B, Koul S, Khandrika L, Meacham RB, Koul HK. Oxidative stress is inherent in prostate cancer cells and is required for aggressive phenotype. Cancer Res. 2008; 68:1777-1785.

72. Clay Montier LL, Deng JJ, Bai Y. Number matters: control of mammalian mitochondrial DNA copy number. J Genet Genomics. 2009; 36:125-131.

73. Xing J, Chen M, Wood CG, Lin J, Spitz MR, Ma J, Amos CI, Shields PG, Benowitz NL, Gu J, de Andrade M, Swan GE, Wu X. Mitochondrial DNA content: its genetic heritability and association with renal cell carcinoma. J Natl Cancer Inst. 2008; 100:1104-1112.

74. Mengel-From J, Thinggaard $\mathrm{M}$, Dalgård $\mathrm{C}$, Kyvik KO, Christensen K, Christiansen L. Mitochondrial DNA copy number in peripheral blood cells declines with age and is associated with general health among elderly. Hum Genet. 2014; 133:1149-1159.

75. Hu L, Yao X, Shen Y. Altered mitochondrial DNA copy number contributes to human cancer risk: evidence from an updated meta-analysis. Sci Rep. 2016; 6:35859.

76. Chen N, Wen S, Sun X, Fang Q, Huang L, Liu S, Li W, Qiu M. Elevated Mitochondrial DNA Copy Number in Peripheral Blood and Tissue Predict the Opposite Outcome of Cancer: A Meta-Analysis. Sci Rep. 2016; 6:37404.

77. Ellinger J, Müller DC, Müller SC, Hauser S, Heukamp LC, von Ruecker A, Bastian PJ, Walgenbach-Brunagel G. Circulating mitochondrial DNA in serum: a universal diagnostic biomarker for patients with urological malignancies. Urol Oncol. 2012; 30:509-515.

78. Zhou W, Zhu M, Gui M, Huang L, Long Z, Wang L, Chen H, Yin Y, Jiang X, Dai Y, Tang Y, He L, Zhong K. Peripheral blood mitochondrial DNA copy number is associated with prostate cancer risk and tumor burden. PLoS One. 2014; 9:e109470.

79. Moore A, Lan Q, Hofmann JN, Liu CS, Cheng WL, Lin TT, Berndt SI. A prospective study of mitochondrial DNA copy number and the risk of prostate cancer. Cancer Causes Control. 2017; 28:529-538.

80. Tu H, Gu J, Meng QH, Kim J, Davis JW, He Y, Wagar EA, Thompson TC, Logothetis CJ, Wu X. Mitochondrial DNA copy number in peripheral blood leukocytes and the 
aggressiveness of localized prostate cancer. Oncotarget. 2015; 6:41988-41996. https://doi.org/10.18632/oncotarget.5889.

81. Mizumachi T, Muskhelishvili L, Naito A, Furusawa J, Fan CY, Siegel ER, Kadlubar FF, Kumar U, Higuchi M. Increased distributional variance of mitochondrial DNA content associated with prostate cancer cells as compared with normal prostate cells. Prostate. 2008; 68:408-417.

82. Koochekpour S, Marlowe T, Singh KK, Attwood K, Chandra D. Reduced mitochondrial DNA content associates with poor prognosis of prostate cancer in African American men. PLoS One. 2013; 8:e74688.

83. Reznik E, Miller ML, Şenbabaoğlu Y, Riaz N, Sarungbam J, Tickoo SK, Al-Ahmadie HA, Lee W, Seshan VE, Hakimi AA, Sander C. Mitochondrial DNA copy number variation across human cancers. Elife. 2016; 5.

84. Zhou CK, Check DP, Lortet-Tieulent J, Laversanne M, Jemal A, Ferlay J, Bray F, Cook MB, Devesa SS. Prostate cancer incidence in 43 populations worldwide: An analysis of time trends overall and by age group. Int J Cancer. 2016; 138:1388-1400.

85. Kibel AS, Ciezki JP, Klein EA, Reddy CA, Lubahn JD, Haslag-Minoff J, Deasy JO, Michalski JM, Kallogjeri D, Piccirillo JF, Rabah DM, Yu C, Kattan MW, et al. Survival among men with clinically localized prostate cancer treated with radical prostatectomy or radiation therapy in the prostate specific antigen era. J Urol. 2012; 187:1259-1265.

86. Mirza M, Griebling TL, Kazer MW. Erectile dysfunction and urinary incontinence after prostate cancer treatment. Semin Oncol Nurs. 2011; 27:278-289.

87. Jones JM, Kohli M, Loprinzi CL. Androgen deprivation therapy-associated vasomotor symptoms. Asian J Androl. 2012; 14:193-197.

88. Hayes JH, Barry MJ. Screening for prostate cancer with the prostate-specific antigen test: a review of current evidence. JAMA. 2014; 311:1143-1149.

89. Heidenreich A, Bastian PJ, Bellmunt J, Bolla M, Joniau S, van der Kwast T, Mason M, Matveev V, Wiegel T, Zattoni F, Mottet N, European Association of Urology. EAU guidelines on prostate cancer. part 1: screening, diagnosis, and local treatment with curative intent-update 2013. Eur Urol. 2014; 65:124-137.

90. Jerónimo C, Nomoto S, Caballero OL, Usadel H, Henrique R, Varzim G, Oliveira J, Lopes C, Fliss MS, Sidransky D. Mitochondrial mutations in early stage prostate cancer and bodily fluids. Oncogene. 2001; 20:5195-5198.

91. Mehra N, Penning M, Maas J, van Daal N, Giles RH, VoestEE. Circulating mitochondrial nucleic acids have prognostic value for survival in patients with advanced prostate cancer. Clin Cancer Res. 2007; 13:421-426.

92. Ellinger J, Müller SC, Wernert N, von Ruecker A, Bastian PJ. Mitochondrial DNA in serum of patients with prostate cancer: a predictor of biochemical recurrence after prostatectomy. BJU Int. 2008; 102:628-632.
93. Wong SQ, Li J, Tan AYC, Vedururu R, Pang JMB, Do H, Ellul J, Doig K, Bell A, MacArthur GA, Fox SB, Thomas DM, Fellowes A, et al. Sequence artefacts in a prospective series of formalin-fixed tumours tested for mutations in hotspot regions by massively parallel sequencing. BMC Med Genomics. 2014; 7:23.

94. Zhang P, Samuels DC, Lehmann B, Stricker T, Pietenpol J, Shyr Y, Guo Y. Mitochondria sequence mapping strategies and practicability of mitochondria variant detection from exome and RNA sequencing data. Brief Bioinformatics. 2015.

95. Álvarez-Cubero MJ, Saiz Guinaldo M, MartínezGonzález LJ, Álvarez Merino JC, Cózar Olmo JM, Acosta JAL. Mitochondrial haplogroups and polymorphisms reveal no association with sporadic prostate cancer in a southern European population. PLoS One. 2012; 7:e41201.

96. Ishikawa K, Koshikawa N, Takenaga K, Nakada K, Hayashi JI. Reversible regulation of metastasis by ROS-generating mtDNA mutations. Mitochondrion. 2008; 8:339-344.

97. Core Team R. R: A language and environment for statistical computing. Vienna, Austria: R Foundation for Statistical Computing; 2016 [cited 2016 Nov 2]. Available from: http://www.R-project.org/.

98. Wickham H. ggplot2. New York, NY: Springer New York; 2009.

99. Kim W, Yoo TK, Shin DJ, Rho HW, Jin HJ, Kim ET, Bae YS. Mitochondrial DNA haplogroup analysis reveals no association between the common genetic lineages and prostate cancer in the Korean population. PLoS One. 2008; 3:e2211.

100. Mueller EE, Eder W, Mayr JA, Paulweber B, Sperl W, Horninger W, Klocker H, Kofler B. Mitochondrial haplogroups and control region polymorphisms are not associated with prostate cancer in Middle European Caucasians. PLoS One. 2009; 4:e6370.

101. Cano D, Gomez CF, Ospina N, Cajigas JA, Groot H, Andrade RE, Torres MM. Mitochondrial DNA haplogroups and susceptibility to prostate cancer in a colombian population. ISRN Oncol. 2014; 2014:530675.

102. Purcell S, Cherny SS, Sham PC. Genetic Power Calculator: design of linkage and association genetic mapping studies of complex traits. Bioinformatics. 2003; 19:149-150.

103. Pisani P, Bray F, Parkin DM. Estimates of the world-wide prevalence of cancer for 25 sites in the adult population. Int J Cancer. 2002; 97:72-81.

104. Gómez-Zaera M, Abril J, González L, Aguiló F, Condom E, Nadal M, Nunes V. Identification of somatic and germline mitochondrial DNA sequence variants in prostate cancer patients. Mutat Res. 2006; 595:42-51.

105. Chen JZ, Gokden N, Greene GF, Mukunyadzi P, Kadlubar FF. Extensive somatic mitochondrial mutations in primary prostate cancer using laser capture microdissection. Cancer Res. 2002; 62:6470-6474. 